\title{
Existence and stability of curved multidimensional detonation fronts
}

\author{
N. Costanzino* H. K. Jenssen† G. Lyng; Mark Williams,
}

August 12, 2006

\begin{abstract}
The rigorous study of spectral stability for ZND detonations was begun by J.J. Erpenbeck in [E1]. He used a normal mode analysis to define a stability function $V(\lambda, \eta)$, whose zeros in $\Re \lambda>0$ correspond to multidimensional perturbations of a steady planar profile that grow exponentially with time. In [E3] he was able to prove that for large classes of steady ZND profiles, unstable zeros of $V$ always exist in the high frequency regime, even when the von Neumann shock, regarded as a gas dynamical shock, is uniformly stable in the sense (later) defined by Majda; subsequent numerical work has shown that unstable zeros usually exist in the medium frequency regime as well.

In this paper we begin a rigorous study of the implications for nonlinear stability of the spectral instabilities just described. We show that in spite of the existence of unstable zeros of $V(\lambda, \eta)$, one can prove the finite (but arbitrarily long) time existence of slightly curved, nonsteady, multidimensional detonation fronts for ideal polytropic gases in both the ZND and Chapman-Jouguet models. In the ZND case we show that this nonlinear stability problem is actually governed by a different stability function, $\Delta_{Z N D}(\hat{\lambda}, \hat{\eta})$, which turns out to coincide with the high frequency limit of $V(\lambda, \eta) /|\lambda, \eta|$ in $\Re \hat{\lambda}>0$. Moreover, the above nonlinear stability result for ideal polytropic gases holds more generally in any situation where $\Delta_{Z N D}(\hat{\lambda}, \hat{\eta})$ is bounded away from zero in $\Re \hat{\lambda}>0$. We also revisit the argument of [E3] in order to simplify and complete some of the analysis in the proof of the main result there.
\end{abstract}

\section{Contents}

${ }^{*}$ UNC Chapel Hill

${ }^{\dagger}$ Penn. State University, partially supported by NSF grants DMS-0206631 and DMS-0539549 (CAREER)

${ }^{\ddagger}$ University of Wyoming, partially supported by the NSF VIGRE program at the University of Michigan (DMS-9977371)

$\S$ UNC Chapel Hill, partially supported by NSF grant DMS-0401252

"We thank Kevin Zumbrun for comments on the manuscript and stimulating conversations related to this work. We also thank the referee for some helpful corrections. 
2 Chapman-Jouguet and ZND fronts $\quad 7$

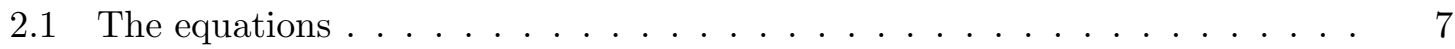

2.2 Steady planar profiles . . . . . . . . . . . . . . . . . . 10

2.3 Curved fronts . . . . . . . . . . . . . . . . . . . 12

3 Assumptions 13

4 Linearization and stability determinants $\quad \mathbf{1 5}$

4.1 The linearized ZND system . . . . . . . . . . . . . . . . . 15

4.2 The linearized Chapman-Jouguet system . . . . . . . . . . . . . . . 17

4.3 The Chapman-Jouguet determinant . . . . . . . . . . . . . . . . . 18

4.4 The ZND Evans function $D_{Z N D}(\zeta) \ldots \ldots \ldots \ldots \ldots \ldots$

4.5 Relation to Erpenbeck's stability function $V(\zeta) \ldots \ldots \ldots \ldots \ldots \ldots$

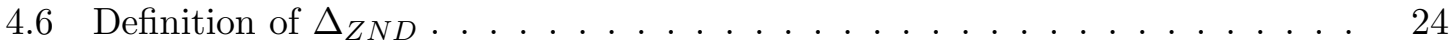

5 The high frequency regime. $\quad \mathbf{2 6}$

5.1 Tracking Lemma and high frequency limit of $D_{Z N D}(\zeta) /|\zeta| \ldots \ldots \ldots$

5.2 Asymptotic behavior as both $x \rightarrow-\infty$ and $|\zeta| \rightarrow \infty \ldots \ldots \ldots$

6 Stability determinants for the physical equations 31

6.1 Computation of $\Delta_{Z N D}$ and $\Delta_{C J} \ldots \ldots \ldots \ldots \ldots \ldots \ldots$

$7 \quad$ Existence of curved ZND detonation fronts $\quad 34$

8 Appendix: Curved fronts in the Chapman-Jouguet model 44

\section{Introduction}

In this paper we study the spectral and nonlinear stability of strong detonations in the two most commonly studied inviscid models of combustion, the Zeldovich-von NeumannDöring (finite reaction rate) and Chapman-Jouguet (instantaneous reaction) models. The rigorous study of spectral stability for ZND detonations was begun by J.J. Erpenbeck in [E1]. He used a normal mode analysis to define a stability function $V(\lambda, \eta)$, whose zeros in $\Re \lambda>0$ correspond to multidimensional perturbations of a steady planar profile that grow exponentially with time. (Here $\lambda \in \mathbb{C}$ is the Laplace transform variable dual to time, and $\eta \in$ $\mathbb{R}^{d-1}$ represents the Fourier transform variables dual to the transverse spatial directions.) The profile in the reaction zone, $x \leq 0$, is given by a nonconstant function, say $\tilde{w}(x)$, of distance from the front, so it is impossible to give an exact, explicit formula for $V(\lambda, \eta)$ from which the unstable zeros, if they exist, can be determined by hand. Numerical computations (e.g., [LS, Sh, SS]) have shown that unstable zeros usually do exist for perturbations in the medium frequency range $\left(\rho_{0} \leq|\lambda, \eta| \leq R\right)$. In a remarkable paper Erpenbeck [E3] was able to show that for large classes of steady ZND profiles, unstable zeros always exist in the high frequency regime, that is, for $|\lambda, \eta| \geq R$ for $R$ arbitrarily large. We shall refer to this result as Erpenbeck's Instability Theorem. An easily computable (by hand) necessary and sufficient condition for the existence of zeros in the low frequency range was given in [JLW]; 
this criterion implies, for example, that for ideal polytropic gases there are no unstable zeros in the range $0 \leq|\lambda, \eta| \leq \rho_{0}$ for $\rho_{0}>0$ sufficiently small.

A striking feature of the Instability Theorem, explained further below, is that $V(\lambda, \eta)$ may have unstable high frequency zeros even when the von Neumann shock, considered as a purely gas dynamical shock, is uniformly stable in the sense of Majda [Ma1].

The goal of this paper is two-fold. First, we revisit the argument of [E3] with the object of simplifying and completing some of the analysis in the proof of the Instability Theorem. The main results of this sort are in section 5. Second, we begin a rigorous study of the implications for nonlinear stability of the spectral instabilities just described. We show in sections 6 and 7 that in spite of the existence of unstable zeros of $V(\lambda, \eta)$, one can prove the finite time existence of curved, nonsteady, multidimensional detonation fronts for ideal polytropic gases (and for other cases satisfying the spectral stability conditions (7.8), (8.3)) in both the ZND and Chapman-Jouguet models. More precisely, given a steady planar ZND profile $\tilde{w}(x)$ discontinuous at $x=0$ and an arbitrarily large but finite time $T_{0}$, for sufficiently small multidimensional perturbations of $\tilde{w}(x)$ at $t=0$ satisfying appropriate corner compatibility conditions, an exact nonsteady solution of the ZND equations, which is discontinuous across a curved front, exists on the time interval $\left[0, T_{0}\right]$. There is a similar result for Chapman-Jouguet fronts. In both cases there is ample time for the disturbance, which travels at finite speed, to move far into the (formerly) steady reaction and quiescent zones. A similar argument [Cos] yields a short-time existence result for larger initial perturbations whose size does not depend on a prescribed $T_{0}$.

An interesting and perhaps surprising conclusion to be drawn from our existence proof is that unstable zeros of $V(\lambda, \eta)$ in the medium frequency range are irrelevant to the question of finite time existence of slightly curved detonation fronts. It is only the high frequency behavior that is important. But why, then, don't the high frequency zeros identified by the Instability Theorem of [E3] rule out such a nonlinear stability result? The answer is provided by the combination of Theorems 5.1 and 7.2. To explain this, first we define polar coordinates

$$
(\lambda, \eta)=\rho(\hat{\lambda}, \hat{\eta}), \text { where }(\hat{\lambda}, \hat{\eta}) \in \bar{S}_{+}^{d}:=\left\{(\lambda, \eta) \in \mathbb{C} \times \mathbb{R}^{d-1}:|\lambda, \eta|=1, \Re \lambda \geq 0\right\}
$$

where $d$ is the number of space dimensions. In section 4.6 we introduce $\Delta_{Z N D}(\hat{\lambda}, \hat{\eta})(4.60)$, a Majda-type determinant [Ma1] defined by linearizing the ZND equations at the piecewise constant von Neumann state, $\left(\tilde{w}\left(0^{-}\right), \tilde{w}\left(0^{+}\right)\right)$, and then throwing away the zero-order reaction forcing term but keeping the first-order part of the reaction equation (Definition 4.60). Theorem 5.1 and Proposition 4.10 imply that

$$
\lim _{\rho \rightarrow \infty} \frac{V(\rho(\hat{\lambda}, \hat{\eta}))}{\rho}=\Delta_{Z N D}(\hat{\lambda}, \hat{\eta}) \text { for } \Re \hat{\lambda}>0
$$

and, furthermore, for any fixed $c>0$ the convergence is uniform on $\bar{S}_{+}^{d} \cap\{\Re \hat{\lambda} \geq c\}$. The importance of $\Delta_{Z N D}$ is further clarified by Theorem 7.2 , which shows that the question of finite time existence of slightly perturbed planar fronts is governed by the determinant $\Delta_{Z N D}$. Since $\Delta_{Z N D}$ is defined by linearization about a piecewise constant object, it is easily computable by hand. In section 6 we give necessary and sufficient conditions for $\Delta_{Z N D}$ to have zeros in $\bar{S}_{+}^{d}$, after showing that it turns out to be a nonvanishing multiple 
of the Majda determinant corresponding to the von Neumann shock considered as a purely gas dynamical shock (that is, as a step shock in a nonreactive gas with the same equation of state as the unburnt gas). Applying these conditions to ideal polytropic gases we find

$$
\left|\Delta_{Z N D}(\hat{\lambda}, \hat{\eta})\right| \geq \delta \text { on } \bar{S}_{+}^{d} \text { for some } \delta>0 \text {. }
$$

In Proposition 7.7 we show that (1.3) is sufficient to imply the $L^{2}$ estimate (7.42) for the ZND equations linearized about a slightly perturbed state (in fact, it is also a necessary condition for (7.42) to hold). This estimate is strong enough to carry out the rest of the nonlinear stability argument in section 7 .

The previous paragraph explains why only the high frequency behavior of $V(\lambda, \eta)$ is important for the nonlinear stability question being studied here; but how do we reconcile (1.3) with (1.2) and the vanishing of $V(\rho(\hat{\lambda}, \hat{\eta}))$, demonstrated by the Instability Theorem, for certain choices of $(\hat{\lambda}, \hat{\eta}, \rho)$ with $\Re \hat{\lambda}>0$ and $\rho$ arbitrarily large? There is no contradiction because in (1.2) we claim that the convergence is uniform only on $\bar{S}_{+}^{d} \cap\{\Re \hat{\lambda} \geq c\}$ for any fixed $c>0$; the Instability Theorem demonstrates that the convergence fails to be uniform on $\bar{S}_{+}^{d} \cap\{\Re \hat{\lambda}>0\}$. The unstable zeros $\left(\hat{\lambda}_{n}, \hat{\eta}_{n}, \rho_{n}\right)$ found by Erpenbeck have the property that

$$
\Re \hat{\lambda}_{n} \downarrow 0 \text { as } \rho_{n} \rightarrow \infty \text {. }
$$

When (1.3) holds, one can regard the zeros $\left(\hat{\lambda}_{n}, \hat{\eta}_{n}, \rho_{n}\right)$ as arising solely from the reactive nature of the gas. Theorem 7.2 shows that when (1.3) holds, these high frequency zeros are also irrelevant to our particular nonlinear stability question.

On the other hand when $\Delta_{Z N D}\left(\hat{\lambda}_{0}, \hat{\eta}_{0}\right)=0$ for some $\Re \hat{\lambda}_{0}>0$ and $\hat{\eta}_{0}$ such that the function

$$
\hat{\lambda} \rightarrow \Delta_{Z N D}\left(\hat{\lambda}, \hat{\eta}_{0}\right) \text { is not identically } 0,
$$

then we can use Rouché's Theorem by virtue of analyticity in $\hat{\lambda}$ to conclude from the uniform limit $(1.2)$ that for $\rho$ sufficiently large $V(\rho(\hat{\lambda}, \hat{\eta}))$ has zeros near $\left(\hat{\lambda}_{0}, \hat{\eta}_{0}\right)$. In this case we cannot prove nonlinear stability as in Theorem 7.2 and we expect it to fail. The $L^{2}$ estimate (7.42), for example, is known to fail in this case. As observed in [Z1], Lemma 3.2, the homogeneity of $\Delta_{Z N D}$ implies that the condition (1.5) holds for all $\eta_{0}$ provided one-dimensional stability holds:

$$
\Delta_{Z N D}(1,0) \neq 0 .
$$

Observe that (1.3) and the uniform convergence in (1.2) imply:

$$
\text { for } \Re \hat{\lambda} \geq c \text {, there exists } R(c) \text { such that } V(\rho(\hat{\lambda}, \hat{\eta})) \neq 0 \text { when } \rho \geq R(c) \text {. }
$$

In fact the proof of (1.2) shows

$$
R(c)=O\left(\frac{1}{c}\right)
$$


A limit of the form (1.2) was proved in [E3], but with the determinant $\Delta_{Z N D}$ replaced by a stability function for nonreactive step shocks, $L_{1}(\hat{\lambda}, \hat{\eta})$, whose definition [E2] is not obviously equivalent to $\Delta_{Z N D}$. The question of uniformity of the limit was not addressed in [E3], so it was not possible there to deduce (1.7) or (1.8) from nonvanishing of $L_{1}$. The proof of (1.2) uses the Tracking Lemma ([Z1], Cor. 8.25) and is simpler than corresponding arguments in [E3]; for example, there is no need to construct approximate solutions or to consider separately different profile types such as those denoted $D, I$, and $M$ in [E3]. However, the hardest step in the proof of the Instability Theorem is to study the behavior of $V$ near $\Re \hat{\lambda}=0$ and, except for the case considered in part (b) of Theorem 5.1, we have not found a way to simplify that part of the argument of [E3].

The importance of having determinant formulations for stability functions like $\Delta_{Z N D}$ (4.60) and $\Delta_{C J}$ (4.23) became quite clear with the work of Kreiss $[\mathrm{K}]$ for hyperbolic fixed boundary problems and Majda [Ma1, Ma2] for free boundary problems. These formulations are similar to the classical Lopatinski determinants defined earlier for elliptic boundary problems. Roughly speaking, such determinants are nonvanishing when the decaying eigenspace of the Fourier-Laplace transformed interior linearized problem (e.g., (4.57)) has trivial intersection with the kernel of the boundary operator. In the hyperbolic setting nonvanishing of such determinants on $\bar{S}_{+}^{d}$, together with the block structure condition [K, Ma1, Met2], are precisely the conditions needed to construct Kreiss symmetrizers, which then yield $L^{2}$ estimates like (7.42). The Majda determinant for the von Neumann shock, considered as a nonreactive shock, can be shown to be a nonvanishing multiple of Erpenbeck's $L_{1}(\hat{\lambda}, \hat{\eta})$; the argument is similar to the proof of Proposition 4.8.

It is also valuable to have an alternative formulation of Erpenbeck's ZND stability function $V(\lambda, \eta)$ as a Lopatinski-type determinant or Evans function; in section 4.4 we define the Evans function $D_{Z N D}(\lambda, \eta)$ (4.37) globally on

$$
\left\{\rho(\hat{\lambda}, \hat{\eta}):(\hat{\lambda}, \hat{\eta}, \rho) \in \bar{S}_{+}^{d} \times(0, \infty)\right\}
$$

show that it is $C^{\infty}$ in $S_{+}^{d} \times(0, \infty)$ with a continuous extension to $\bar{S}_{+}^{d} \times(0, \infty)$ (Cor. 4.5), and show that $D_{Z N D}$ is a nonvanishing multiple of $V$ (Prop. 4.10). A definition of $D_{Z N D}$ as a $C^{\infty}$ function on $S_{+}^{d} \times\left(0, \rho_{0}\right]$ for $\rho_{0}$ small, together with a proof of the continuous extendability to $\bar{S}_{+}^{d} \times\left[0, \rho_{0}\right]$ was given in [JLW]. When $\rho$ is small, the real parts of the eigenvalues of the lower right "reaction block" in $\mathcal{G}\left(w_{-}, \lambda, \eta\right)(4.25)$ are strictly greater than and bounded away from the real parts of the eigenvalues of the upper left "gas dynamical" block. This is no longer true for $\rho$ large, where one has crossing of $\Re \mu_{j}$ for eigenvalues $\mu_{j}$ associated to different blocks. Since this crossing can happen when $\Re \hat{\lambda}=0$, some care is needed in order to obtain a continuous extension of $D_{Z N D}$ (or $V$ ) to $\Re \hat{\lambda} \geq 0$ when $\rho$ is large (Prop. 4.4).

Each of the two formulations of the ZND stability function, $D_{Z N D}$ (4.37) and $V$ (4.54) has its advantages. The determinant form leads more directly to $L^{2}$ estimates as indicated above, and is convenient for low-frequency analysis [JLW], while Erpenbeck's form is better from the point of view of numerical computations. In proving the connection between $D_{Z N D}$ and $V$ we give a third formulation, $\mathbb{D}_{Z N D}$ (4.48), which is similar to $V$ but even better for numerical computations (see $[\mathrm{HZ}]$ ). This form is also the most convenient for proving the high frequency limit (1.2). The simpler form of $\mathbb{D}_{Z N D}$ arises from the use of the "good 
unknown" $w^{\#}$ (4.6) introduced in [JLW], which eliminates the forcing terms depending on the front in the interior linearized ZND equations (compare (4.7) and (4.5)).

Section 5.2 is included in order to complete an argument in the proof of the Instability Theorem of [E3]. The interior linearized ZND equations can be written as the first order system of ODEs (4.13) depending on parameters $(\lambda, \eta)$

$$
\partial_{x} w^{\#}=\mathcal{G}(\tilde{w}(x), \lambda, \eta) w^{\#} \text { in } \pm x>0,
$$

where $w^{\#}$ is the "good unknown" chosen in (4.6) to simplify the form of the equations. The proof of the Instability Theorem of [E3], like that of the high frequency limit (1.2), is based on understanding the behavior as $\rho \rightarrow \infty$ of the unique (up to constant multiple) solution $\tilde{\theta}(x, \lambda, \eta)(5.16)$ of the transposed system

$$
\partial_{x} \tilde{\theta}=-\mathcal{G}^{t}(\tilde{w}(x), \lambda, \eta) \tilde{\theta}
$$

that decays to zero as $x \rightarrow-\infty$ when $\Re \lambda>0$. Erpenbeck's argument, roughly rephrased in our notation, is based on constructing an approximate WKB-type solution $\tilde{\theta}_{a}(x, \rho(\hat{\lambda}, \hat{\eta}))$ to (1.11) that stays close as $\rho \rightarrow \infty$ to the exact solution $\tilde{\theta}(x, \rho(\hat{\lambda}, \hat{\eta}))$ for all $x \leq 0$, except for a finite set of "turning points" $x_{j}^{*}(\hat{\lambda}, \hat{\eta}, \rho)<0, j=1, \ldots, N$, where $x_{1}^{*}$ represents the leftmost point. A preliminary step, whose purpose is to replace $(-\infty, 0]$ with a bounded interval $\left[x_{0}^{*}(\hat{\lambda}, \hat{\eta}, \rho), 0\right]$, where $x_{0}^{*}<x_{1}^{*}$, is carried out at the beginning of section III of [E3]. The idea is to choose $\left|x_{0}^{*}\right|$ large enough so that $\tilde{\theta}(x, \lambda, \eta)$ remains "close" (see (1.15)), on the interval

$$
\left(-\infty, x_{0}^{*}(\hat{\lambda}, \hat{\eta}, \rho)\right]
$$

to a decaying solution $\theta_{L}(x, \lambda, \eta)$ of the constant-coefficient limiting problem obtained from (1.11) by letting $x \rightarrow-\infty$ :

$$
\partial_{x} \theta_{L}=-\mathcal{G}^{t}\left(w_{-}, \lambda, \eta\right) \theta_{L}
$$

Friedrichs's method of the parameter problem is then applied to justify the use of $\tilde{\theta}_{a}$ as an approximation to $\tilde{\theta}$ on the bounded interval that remains. A potentially serious difficulty is that $x_{0}^{*}$ with these properties depends on $(\hat{\lambda}, \hat{\eta}, \rho)$ and, generally,

$$
x_{0}^{*}(\hat{\lambda}, \hat{\eta}, \rho) \rightarrow-\infty \text { as } \rho \rightarrow \infty .
$$

It is important to estimate the rate of growth of $\left|x_{0}^{*}\right|$ in (1.14) and to understand how that rate depends on $(\hat{\lambda}, \hat{\eta})$. For example, exponential growth would invalidate the entire argument of [E3]. A growth rate of $\rho^{N}$ would necessitate the use of $\sim N$ terms in the WKB expansion of $\tilde{\theta}_{a}$. These points were not addressed in [E3], so we consider them in section 5.2. In Proposition 5.7 we show that given any $\epsilon>0$, it is possible to choose $x_{0}^{*}$ so that

$$
\left|\tilde{\theta}(x, \lambda, \eta)-\theta_{L}(x, \lambda, \eta)\right| e^{-\mu_{+}(\lambda, \eta) x} \leq \epsilon \quad \text { for } x \leq x_{0}^{*}(\hat{\lambda}, \hat{\eta}, \rho),(\hat{\lambda}, \hat{\eta}) \in \bar{S}_{+}^{d}, \rho \geq 2,
$$

where

$$
\left|x_{0}^{*}(\hat{\lambda}, \hat{\eta}, \rho)\right| \leq C \ln \rho \text { for } C \text { independent of }(\hat{\lambda}, \hat{\eta}) \in \bar{S}_{+}^{d}, \rho \geq 2
$$


The proof of Proposition 5.7 is a modification of the proof of the Gap Lemma of [Z1]. It takes advantage both of the exponential rate of convergence of $\tilde{w}(x)$ to $w_{-}$and of the fact that the rate of decay of $\tilde{\theta}(x, \lambda, \eta)$ as $x \rightarrow-\infty$ is governed by an extreme eigenvalue $\mu_{+}(\lambda, \eta)$ of $-\mathcal{G}^{t}\left(w_{-}, \lambda, \eta\right)$. As far as we know, this is the first application of Gap Lemmatype arguments to investigate asymptotic behavior in $x$ as a parameter like frequency tends to infinity.

The existence proofs in section 7 and the Appendix have much in common with the arguments of [Ma1, Ma2]. A few of the differences are:

(a) Majda's result was a short-time result; in Theorems 7.2 and 8.1 we fix an arbitrarily large but finite time $T_{0}$ and show that slightly curved fronts exist on the time interval $\left[0, T_{0}\right]$.

(b) In the iteration scheme for the boundary conditions, we do not make use of Newton's method; we simply use (7.25) where $\mathcal{B}$ is defined in (7.23).

(c) In the case of Chapman-Jouguet fronts, there is a new complication due to the fact that the definition of the fluxes $\tilde{f}^{j}$ in (2.30) changes discontinuously across the free surface given by the curved front. For ZND there is a complication due to crossing of eigenvalues associated to gas dynamical and reaction blocks. These points affect the verification of the block structure and uniform Lopatinski conditions.

It would be interesting to compare the high frequency behavior of the stability function for the full reactive Navier-Stokes equations, $D_{R N S}(\lambda, \eta)$, defined in [JLW], to the behavior of $\Delta_{Z N D}$ and $D_{Z N D}$. These equations contain extra second-order terms corresponding to viscosity, heat conduction, and species diffusion. Does $D_{R N S}$ have high frequency zeros like those of $D_{Z N D}$ ? The Equivalence Theorem of [JLW] shows that the low frequency behavior of $D_{R N S}$ is identical to that of $D_{Z N D}$ and governed by $\Delta_{C J}$. Thus, for example, nonvanishing of $\Delta_{C J}(\hat{\lambda}, \hat{\eta})$ on $\bar{S}_{+}^{d}$ implies the complete absence of low frequency zeros for both $D_{R N S}(\lambda, \eta)$ and $D_{Z N D}(\lambda, \eta)$.

Finally, we wish to state a few open nonlinear stability questions for which we would expect any unstable zeros of $V(\lambda, \eta)$ to present significant difficulties:

1. Construct curved multidimensional ZND fronts that converge to curved multidimensional Chapman-Jouguet fronts in the limit as reaction rate $(k$ in $(2.1 \mathrm{~d}))$ tends to infinity.

2. Construct smooth solutions to the full reactive Navier-Stokes equations which converge to curved multidimensional ZND fronts as the coefficients of the second-order terms tend to zero.

3. Study the long-time stability $\left(T_{0} \rightarrow \infty\right)$ of reactive Navier-Stokes profiles. Here there are dissipative effects that provide some mechanism for decay of perturbations.

\section{Chapman-Jouguet and ZND fronts}

\subsection{The equations}

We set $y_{d}=x$ and denote spatial directions by $\left(y_{1}, \ldots, y_{d}\right)$. The Zeldovich-von NeumannDöring (ZND) equations for a $d$-dimensional reacting fluid with a one-step exothermic reaction are given in Eulerian coordinates as (see, e.g., [Wi])

$$
\begin{aligned}
\rho_{t}+\operatorname{div}(\rho \mathbf{u}) & =0, \\
\left(\rho u_{j}\right)_{t}+\operatorname{div}\left(\rho u_{j} \mathbf{u}\right)+p_{y_{j}} & =0, \quad j=1, \ldots d,
\end{aligned}
$$




$$
\begin{gathered}
(\rho \tilde{E})_{t}+\operatorname{div}[(\rho \tilde{E}+p) \mathbf{u}]=0, \\
(\rho Y)_{t}+\operatorname{div}(\rho Y \mathbf{u})=-k \rho Y \phi(T) .
\end{gathered}
$$

Here the unknowns are $(\rho, \mathbf{u}, T, Y)$ and the system has dimension $(n+s) \times(n+s)$, where

$$
n=d+2, s=1 \text {. }
$$

(Later we'll consider a more complicated multi-step model in which several species of gas are involved, so in that case $s>1$.)

The divergence is taken with respect to the spatial directions. We write

$$
\tilde{E}=\tilde{e}+\frac{|\mathbf{u}|^{2}}{2},
$$

and our labels are given in the table below.

\begin{tabular}{|ll|}
\hline$\rho$ & density \\
$p$ & pressure \\
$\mathbf{u}=\left(u_{1}, \ldots, u_{d}\right)^{t}$ & fluid velocity \\
$T$ & temperature \\
$\tilde{e}$ & specific internal energy \\
$Y$ & mass fraction of reactant \\
\hline$k$ & reaction rate \\
$q$ & heat release \\
\hline
\end{tabular}

The quantities $k$ and $q$ are assumed to be positive constants. We note that the assumption $q>0$ corresponds to an exothermic reaction. The internal energy of the mixture of burnt and unburnt gas is given by (see $[\mathrm{Ma} 3, \mathrm{FD}]$, e.g.)

$$
\tilde{e}=e+q Y, Y \in[0,1]
$$

where $e$ is the internal energy of the completely burnt gas, and we set $E:=e+|\mathbf{u}|^{2} / 2$. We further assume that $p=p(\rho, T)$ and $e=e(\rho, T)$ are given functions of density and temperature satisfying

$$
p_{\rho}>0, e_{T}>0
$$

In addition we suppose $p, e$, and the entropy $s=s(\rho, T)$ of the completely burnt gas satisfy the thermodynamic relation

$$
d e=T d s-p d v, \text { where } v=1 / \rho .
$$

Remark 2.1. The thermodynamic variables can also be regarded as functions of $(\rho, s)$, which we denote $\hat{p}(\rho, s), \hat{e}(\rho, s)$, etc.. Given (2.5), the condition (2.4) is equivalent to the statement that $e$ is a convex function of $(v, s)$ (thermodynamic stability; [Z2]). In turn this immediately implies $\hat{p}_{\rho}(\rho, s)>0$. The square of the sound speed in the completely burnt gas is given by

$$
c^{2}=\hat{p}_{\rho}(\rho, s) \quad([C F]) .
$$


Finally, the smooth, increasing function function $\phi(T)$ is the ignition function. We make the standard assumption that $\phi$ satisfies ignition temperature kinetics, that is,

$$
\phi(T)=\left\{\begin{array}{l}
0, \text { for } T<T_{i} \\
1, \text { for } T>T_{0}>T_{i}
\end{array} .\right.
$$

Thus, $\phi$ serves to turn on the reaction in equation (2.1d).

Subtracting $q \cdot(2.1 \mathrm{~d})$ from equation $(2.1 \mathrm{c})$ we obtain,

$$
(\rho E)_{t}+\operatorname{div}[(\rho E+p) \mathbf{u}]=q k \rho Y \phi(T) .
$$

If we denote the gas-dynamical variables by $V=(\rho, \mathbf{u}, T)$, and set

$$
w=(V, Y), V \in \mathbb{R}^{n}, Y \in \mathbb{R}^{s}
$$

and $y_{0}=t$, we see that the system $(2.1 \mathrm{a}),(2.1 \mathrm{~b}),(2.8),(2.1 \mathrm{~d})$ is a special case of the following abstract model corresponding to an $s$-step exothermic reaction:

$$
\sum_{j=0}^{d} F^{j}(w)_{y_{j}}=R(w) .
$$

Here, corresponding to the decomposition $w=(V, Y)$, we have

$$
F^{j}(w)=\left(\begin{array}{c}
f^{j}(V) \\
g^{j}(V) Y
\end{array}\right), j=0, \ldots, d,
$$

where $f^{j} \in \mathbb{R}^{n}, g^{j} \in \mathbb{R}^{1}$. The forcing term is

$$
R(w)=\left(\begin{array}{l}
Q K \psi(V) Y \\
-K \psi(V) Y
\end{array}\right)
$$

where $\psi \in \mathbb{R}^{1}, Q \in \mathbb{R}^{n \times s}$ and $K \in \mathbb{R}^{s \times s}$ are constant matrices, and

(a) $K$ is positive definite,

(b) the first $n-1$ rows of $Q$ are 0 .

For the one-step physical equations we have, for example,

$$
\begin{aligned}
& g^{0}(V)=\rho, g^{j}(V)=\rho u_{j}, j=1, \ldots, d \\
& \psi(V)=\rho \phi(T) \\
& f^{0}(V)=\left(\begin{array}{lll}
\rho & \rho \mathbf{u} & \rho\left(e+\frac{|\mathbf{u}|^{2}}{2}\right)
\end{array}\right)^{t}, \\
& Q=(0, \ldots, 0, q)^{t} \text {, }
\end{aligned}
$$

where $c_{v}$ is specific heat at constant volume. 
Remark 2.2. In an s-step reaction, the $y_{j}$ component of $Y \in \mathbb{R}^{s}$ represents the mass fraction of the $j$-th reactant, with $y_{j} \in[0,1]$ and $y_{j}=1$ (resp. 0) corresponding to the completely unburnt (resp., burnt) state. The completely burnt and unburnt states are represented by

$$
\mathbf{0}=(0, \ldots, 0) \in \mathbb{R}^{s}, \mathbf{1}=(1, \ldots, 1) \in \mathbb{R}^{s},
$$

respectively. The $n \times s$ matrix $Q$ in this case has the form

$$
Q=\left(\begin{array}{ccc}
0 & \ldots & 0 \\
\vdots & \ldots & \vdots \\
0 & \ldots & 0 \\
q_{1} & \ldots & q_{s}
\end{array}\right)
$$

where $q_{j}$ denotes the heat released in the $j$ th reaction, $q_{j}>0$ in the case of an exothermic reaction.

Definition 2.3. To obtain the Chapman-Jouguet (CJ) equations from the ZND equations, we eliminate the reaction equation (2.1d), and in the energy equation (2.1c) define $\tilde{e}=e+q$ in the unburned gas (where $Y=1$ ) and $\tilde{e}=e$ in the burnt gas $(Y=0)$.

The corresponding abstract CJ model is

$$
\sum_{j=0}^{d} \tilde{f}^{j}(V)_{y_{j}}=0
$$

where (recall (2.1a)-(2.1d))

$$
\tilde{f}^{j}(V)=\left\{\begin{array}{l}
f^{j}(V), \text { in the burnt gas } \\
f^{j}(V)+g^{j}(V) Q \mathbf{1}, \text { in the unburnt gas }
\end{array}\right.
$$

\subsection{Steady planar profiles}

\subsubsection{The CJ profile.}

We consider a steady solution for the CJ system (2.16)

$$
\tilde{v}=\left\{\begin{array}{l}
V_{+}, x>0 \\
V_{-}, x<0
\end{array}\right.
$$

corresponding to a pair of constant states

$$
w_{+}=\left(V_{+}, \mathbf{1}\right) \text { in } x>0, w_{-}=\left(V_{-}, \mathbf{0}\right) \text { in } x<0,
$$

and satisfying the Rankine-Hugoniot condition at $x=0$ (which expresses conservation of mass, momentum, and energy for the physical equations):

$$
f^{d}\left(V_{+}\right)+g^{d}\left(V_{+}\right) Q \mathbf{1}=f^{d}\left(V_{-}\right) .
$$

We assume that $w_{ \pm}$define a strong detonation with Lax n-shock structure. 
Definition 2.4. Let $a^{j}=d f^{j} \in \mathbb{R}^{n \times n}$, and set $\mathcal{A}^{j}=\left(a^{0}\right)^{-1} a^{j}$. The states $w_{ \pm}$are a strong detonation with Lax $n$-shock structure provided:

(a) they satisfy the jump condition (2.20), and

(b) the $n \times n$ matrix $\mathcal{A}^{d}\left(V_{+}\right)$has $n$ eigenvalues $<0$, while $\mathcal{A}^{d}\left(V_{-}\right)$has $n-1$ eigenvalues $<0$ and one eigenvalue $>0$.

Remark 2.5. 1. The existence of CJ solutions as in Definition 2.4 for the physical equations is proved in $[C F, F D, G S]$. Small amplitude $\left(\left|V_{+}-V_{-}\right|\right)$conditions are not relevant here; the only issue is to obtain the right sort of intersection between the Rayleigh line and the burnt Hugoniot curve. When $q$ is positive, zero amplitude $\left(V_{+}=V_{-}\right)$CJ fronts are impossible, since the burned Hugoniot curve is shifted away from the unburned state. On the other hand, provided suitable relations hold, for example, between the components of the unburnt state $V_{-}$and the heat release q, CJ fronts of arbitrary strength are possible. We assume the existence of CJ solutions for the abstract model.

2. The case of a planar front moving with nonzero constant velocity can be reduced to the present case by a change of frame.

3. In the case of the physical equations, say when $d=3$, the eigenvalues of $\mathcal{A}^{d}(V)$ are (recall $V=(\rho, \mathbf{u}, T)) u_{d}, u_{d}, u_{d}, u_{d} \pm c$, where $c$ is sound speed. Thus, the assumption of $n$-shock structure corresponds to the statement that unburnt gas is moving from right to left across the front $\left(u_{d+}<0\right)$, and that the gas speed is supersonic ahead of the front $\left(\left|u_{d+}\right|>c_{+}\right)$and subsonic behind $\left(\left|u_{d_{-}}\right|<c_{-}\right)$.

\subsubsection{The ZND profile.}

The ZND profile $\tilde{w}(x)=(\tilde{V}(x), \tilde{Y}(x))$ is a weak solution of the ZND abstract model $(2.10)$ which satisfies

$$
\begin{aligned}
& \tilde{w}(x)=w_{+} \text {in } x>0 \\
& F^{d}(\tilde{w})^{\prime}=R(\tilde{w}) \text { in } x<0, \tilde{w} \rightarrow w_{-} \text {as } x \rightarrow-\infty
\end{aligned}
$$

and the jump condition at $x=0$

$$
\left[F^{d}(\tilde{w})\right]_{*}=0 .
$$

Here we let $w^{*}=\left(V^{*}, Y^{*}\right)$ denote the von Neumann state $\tilde{w}\left(0^{-}\right)$just to the left of the discontinuity, and

$$
\left[F^{d}(\tilde{w})\right]_{*}=F^{d}\left(w_{+}\right)-F^{d}\left(w^{*}\right) .
$$

The discontinuity at $x=0$ is referred to as the von Neumann shock.

Remark 2.6. 1. In the case of the physical ZND equations we make the standard assumption that the reaction rate is zero ahead of the shock (i.e., in $x>0$ ) and finite behind. Thus, we assume that the $T$ components of $w_{ \pm}$satisfy

$$
T_{+}<T_{i}, T_{-}>T_{0}
$$

for temperatures $T_{i}, T_{0}$ as in (2.7). 
2. Steady profiles for the physical ZND equations are constructed in [FD, LS, GS, GS2]. In the case of a one-step ideal gas reaction, for example, the reduced phase space for the ZND profile equation is one-dimensional, so the restrictions on which connections are possible are similar to those in the CJ case. Again, smallness conditions are irrelevant. For more complicated multi-step reactions, the reduced phase space is higher dimensional; [GS2] gives an example of a multi-step chain-branching reaction for which there are still connections. We simply assume the existence of ZND profiles for the abstract model.

\subsection{Curved fronts}

Perturbations cause planar fronts to curve, and the perturbed solutions are no longer travelling waves in the above sense. In this section we formulate the hyperbolic free boundary problems that must be solved in order to construct curved detonation fronts.

Suppose the ZND front is a surface $\mathcal{S}$ defined by:

$$
x=X\left(t, y^{\prime}\right), y^{\prime}=\left(y_{1}, \ldots, y_{d-1}\right) .
$$

A $Z N D$ solution $w\left(t, y^{\prime}, x\right)$ is discontinuous across $\mathcal{S}$, satisfies the ZND system

$$
\sum_{j=0}^{d} F^{j}(w)_{y_{j}}=R(w)
$$

on each side of $\mathcal{S}$, and satisfies the jump condition

$$
\sum_{j=0}^{d-1} X_{y_{j}}\left[F^{j}(w)\right]_{*}-\left[F^{d}(w)\right]_{*}=0 \text { on } \mathcal{S} .
$$

(recall $t=y_{0}, x=y_{d}$ ). Given a steady ZND profile $\tilde{w}(x)$ as in $(2.21),(2.22)$ one can try to construct a perturbed solution $w\left(t, y^{\prime}, x\right)$ corresponding to an unknown curved front $x=X\left(t, y^{\prime}\right)$ in the form

$$
w\left(t, y^{\prime}, x\right)=\tilde{w}(x)+W\left(t, y^{\prime}, x\right) .
$$

The $Z N D$ initial boundary value problem for the unknowns $(W, X)$ is to find $X$ and $w$ of the form (2.28) satisfying (2.26), (2.27), and

$$
W\left(0, y^{\prime}, x\right)=v_{0}\left(y^{\prime}, x\right), \quad X\left(0, y^{\prime}\right)=0
$$

where $v_{0}\left(y^{\prime}, x\right)$ is a suitable initial perturbation. The functions $W$ and $X$ are coupled through the jump condition, so the problem defined by $(2.26),(2.27)$ is a free boundary problem for the unknowns $(W, X)$.

Similarly, if we now let $\mathcal{S}$ denote a CJ front, a $C J$ solution $V\left(t, y^{\prime}, x\right)$ satisfies the CJ system

$$
\sum_{j=0}^{d} \tilde{f}^{j}(V)_{y_{j}}=0
$$


on each side and the jump condition

$$
\sum_{j=0}^{d-1} X_{y_{j}}\left[\tilde{f}^{j}(V)\right]-\left[\tilde{f}^{d}(V)\right]=0 \text { on } \mathcal{S} .
$$

where

$$
\tilde{f}^{j}(V)=\left\{\begin{array}{l}
f^{j}(V), \text { for } x<X\left(t, y^{\prime}\right) \\
f^{j}(V)+g^{j}(V) Q \mathbf{1}, \text { for } x>X\left(t, y^{\prime}\right)
\end{array} .\right.
$$

Given a piecewise constant CJ solution $\tilde{v}$ as in (2.18), the CJ initial boundary value problem for the unknowns $(\mathcal{V}, X)$ is to find $X\left(t, y^{\prime}\right)$ and $V$ of the form

$$
V\left(t, y^{\prime}, x\right)=\tilde{v}+\mathcal{V}\left(t, y^{\prime}, x\right)
$$

satisfying $(2.30),(2.31)$, and

$$
\mathcal{V}\left(0, y^{\prime}, x\right)=v_{0}\left(y^{\prime}, x\right), X\left(0, y^{\prime}\right)=0
$$

for a suitable initial perturbation $v_{0}$.

Remark 2.7. Observe that in the Chapman-Jouguet problem (2.30), it is not only the state $V$ that jumps across the curved front, but also the definition of the fluxes $\tilde{f}^{j}(V)$. In the ZND problem (2.26) it is only the state $w$ that jumps.

\section{Assumptions}

For convenient reference we collect here all the structural and profile assumptions that apply to our abstract models. Each of these assumptions is satisfied by the physical CJ and ZND systems.

Notation 3.1. 1. For $F^{j}(w)$ as in (2.11), let

$$
A^{j}(w)=d_{w} F^{j}(w)=\left(\begin{array}{cc}
d f^{j}(V) & 0 \\
d_{V} g^{j}(V) Y & g^{j}(V) I_{s \times s}
\end{array}\right) \in \mathbb{R}^{(n+s) \times(n+s)}
$$

and set $\bar{A}^{j}=\left(A^{0}\right)^{-1} A^{j} \quad$ (see (H1) below). Given a scalar function $h(V)$ and $v \in \mathbb{C}^{n}$, we'll often write

$$
d_{V} h(V) Y v:=\left(d_{V} h(V) \cdot v\right) Y .
$$

2. Let

$$
d f^{j}(V)=a^{j}(V) \in \mathbb{R}^{n \times n}
$$

Similarly, set $d \tilde{f}^{j}=\tilde{a}^{j}$ for $\tilde{f}^{j}$ as in $(2.32)$. 
Assumption 3.1. (HO) The states $w_{ \pm}$define a strong detonation with Lax $n$-shock structure (recall Definition 2.4). The ZND profile decays exponentially to its left endstate with all derivatives: there exists a $\delta>0$ such that for all $k$

$$
\left|(d / d x)^{k}\left(\tilde{w}-w_{-}\right)(x)\right| \leq C_{k} e^{-\delta|x|} \text { as } x \rightarrow-\infty .
$$

In addition, there exists a $C^{\infty}$ invertible matrix $M(V)$ defined in a neighborhood of $V_{+}$, such that, to the right of the front,

$$
\tilde{a}^{j}(V)=M(V) a^{j}(V), j=0, \ldots, d .
$$

Assumption 3.2. There exists an open set $\mathcal{U} \subset \mathbb{R}^{n+s}$ such that the endstates and profile w satisfy:

$$
w_{ \pm} \in \mathcal{U}, \tilde{w}(x) \in \mathcal{U} \text { for all } x
$$

and:

(H1) The functions $F^{j}(w)(2.11)$ and $R(w)$ (2.12) are defined and $C^{\infty}$ in $\mathcal{U}$. The matrix $A^{0}(w)$ is invertible in $\mathcal{U}$ and $g^{0}(V)>C>0$ in $\mathcal{U}$. Let $\mathcal{U}^{-}$be the component of $\mathcal{U}$ containing $\{\tilde{w}(x): x \leq 0\}$. The matrix $A^{d}(w)$ is invertible in $\mathcal{U}^{-}$.

The functions $g^{j}(V)(2.11)$ satisfy the following conditions along the profile:

$$
g^{j}(\tilde{V})=0, j=1, \ldots, d-1 ; \quad g^{d}(\tilde{V})<-\theta<0
$$

for some fixed $\theta>0$.

(H2) The constant matrix $K \in \mathbb{R}^{s \times s}$ (2.12) is positive definite; the constant matrix $Q \in \mathbb{R}^{n \times s}(2.12)$ has its first $(n-1)$ rows equal to 0 ; the function $\psi(V)$ in $(2.12)$ satisfies $\psi\left(V_{+}\right)=0, \psi\left(V_{-}\right)=1, d \psi\left(V_{+}\right)=0$.

Assumption 3.3. (H3) (hyperbolicity) For all $w \in \mathcal{U}$ and $\xi \in \mathbb{R}^{d} \backslash 0$ the eigenvalues of $\bar{A}(w, \xi):=\sum_{j=1}^{d} \bar{A}^{j}(w) \xi_{j}$ are real and semisimple with constant multiplicity.

(H4) (Friedrichs symmetrizability) There exists a $C^{\infty}$ invertible matrix $S(w)$ defined on $\mathcal{U}$ such that $S A^{0}$ is positive definite and for all $j, S A^{j}$ is symmetric. The same holds for the $a^{j}$.

Remark 3.2. 1. In (H3) semisimple means that algebraic and geometric multiplicities are equal.

2. Hypotheses (H0), (H1), and (H2) are straightforward to check for the physical equations. For (HO) see Remark 2.5 and use standard ODE results for the exponential decay (3.4). Note that in the case of an s-step reaction, (3.5) holds since, to the right of the front,

$$
\tilde{a}^{j}(V)=M a^{j}(V) \text {, where } M=\left(\begin{array}{cccccc}
1 & 0 & \cdots & 0 & 0 \\
0 & 1 & \cdots & 0 & 0 \\
0 & 0 & \cdots & 1 & 0 \\
\sum_{j=1}^{s} q_{j} & 0 & \cdots & 0 & 1
\end{array}\right) .
$$

This is easily checked using the fact that $g^{j}(V)$ in (2.14) is equal to the first component of $f^{j}(V)$. One important consequence of (3.5), used in the proof of Theorem 8.1, is

$$
\left(\tilde{a}^{0}\right)^{-1} \tilde{a}^{j}=\left(a^{0}\right)^{-1} a^{j}, j=0, \ldots, d .
$$


To check (3.7) recall Remark 2.5. We can choose $\mathcal{U}$ so that $A^{d}(w)$ is nonsingular in $\mathcal{U}^{-}$, since in the steady flow gas moves from right to left in the reaction zone and at subsonic speed. (H2) holds provided we normalize $\rho_{-}=1$.

Hypotheses (HO) and (H1) imply that the matrix $\bar{A}^{d}\left(w_{+}\right)$has $n+s$ eigenvalues $<0$, while $\bar{A}^{d}(\tilde{w}(x))$ for $x \leq 0$ has one eigenvalue $>0$ and $n+s-1$ eigenvalues $<0$.

3. Using (2.14) and the structure of $\left(A^{0}\right)^{-1}$, hypothesis (H3) may be deduced from the corresponding property in the nonreactive case, i.e., from hyperbolicity of the Euler equations. A Friedrichs symmetrizer $S(w)$ as in (H4) was explicitly given for the physical equations in the appendix of [JLW]. The upper left $n \times n$ block of $S(w)$ is a Friedrichs symmetrizer for the $a^{j}$.

4. Entropy-entropy flux pairs $\left(\eta, q^{j}\right)$ with

$$
\eta=\rho\left(\frac{1}{2} Y^{2}-s\right), q^{j}=\eta u^{j}
$$

where $s$ is the thermodynamic entropy of the unburnt gas, are constructed for the ZND equations in [Cos]. The entropy $\eta$ can be used to construct a Friedrichs symmetrizer.

\section{Linearization and stability determinants}

\subsection{The linearized ZND system}

The ZND system $(2.26),(2.27)$ is a free boundary problem for the unknowns $(w, X)$. First we reduce to a fixed boundary problem by changing coordinates

$$
\left(t, y^{\prime}, x^{*}\right)=\left(t, y^{\prime}, x-X\left(t, y^{\prime}\right)\right), w^{*}\left(t, y^{\prime}, x^{*}\right)=w\left(t, y^{\prime}, x\right) .
$$

With stars dropped, the problem in the new coordinates is

$$
\begin{aligned}
& \sum_{j=0}^{d-1} F^{j}(w)_{y_{j}}+\mathcal{F}^{d}(w, d X)_{x}=R(w) \\
& \sum_{j=0}^{d-1} X_{y_{j}}\left[F^{j}(w)\right]_{*}-\left[F^{d}(w)\right]_{*}=0 \text { on } x=0,
\end{aligned}
$$

where

$$
\mathcal{F}^{d}(w, d X)=F^{d}(w)-\sum_{j=0}^{d-1} X_{y_{j}} F^{j}(w) .
$$

The problem (4.2) can be viewed as a transmission problem for unknowns $\left(w_{ \pm}\left(t, y^{\prime}, x\right), X\left(t, y^{\prime}\right)\right)$ in $\pm x \geq 0$, with transmission conditions given by the jump condition on $x=0$. We'll usually suppress the \pm on $w$ (partly to avoid confusion with the endstates $w_{ \pm}$). 
Linearizing (4.2) with respect to both $w$ and $X$ about the stationary solution given by the ZND profile $\tilde{w}(x)$ and front $X=0$, we obtain

$$
\begin{aligned}
& \sum_{j=0}^{d-1} A^{j}(\tilde{w})\left(\partial_{y_{j}} w-X_{y_{j}} \tilde{w}^{\prime}\right)+\left(A^{d}(\tilde{w}) w\right)^{\prime}=d_{w} R(\tilde{w}) w \\
& \sum_{j=0}^{d-1} X_{y_{j}}\left[F^{j}(\tilde{w})\right]_{*}-\left[A^{d}(\tilde{w}) w\right]_{*}=0 \text { on } x=0
\end{aligned}
$$

where $w$ and $X$ now denote perturbations. Fourier-Laplace transformation gives (dropping hats on $w$ and $X$ )

$$
\begin{aligned}
& \lambda A^{0}(\tilde{w})\left(w-X \tilde{w}^{\prime}\right)+\sum_{j=1}^{d-1} i \eta_{j} A^{j}(\tilde{w})\left(w-X \tilde{w}^{\prime}\right)+\left(A^{d}(\tilde{w}) w\right)^{\prime}=d_{w} R(\tilde{w}) w \\
& X\left(\lambda\left[F^{0}(\tilde{w})\right]_{*}+\sum_{j=1}^{d-1} i \eta_{j}\left[F^{j}(\tilde{w})\right]_{*}\right)-\left[A^{d}(\tilde{w}) w\right]_{*}=0 \text { on } x=0 .
\end{aligned}
$$

Here $\tau \in \mathbb{R}, \eta \in \mathbb{R}^{d-1}, \gamma \geq 0$, and $\lambda=i \tau+\gamma$. For the stability analysis it is convenient to eliminate $X$ from the interior equation by defining new unknowns ( \pm suppressed)

$$
w^{\#}=w-X \tilde{w}^{\prime} .
$$

This gives the equivalent problem

$$
\begin{aligned}
& \text { (a) }\left(\lambda A^{0}(\tilde{w})+\sum_{j=1}^{d-1} i \eta_{j} A^{j}(\tilde{w})\right) w^{\#}+\left(A^{d}(\tilde{w}) w^{\#}\right)^{\prime}=d_{w} R(\tilde{w}) w^{\#} \\
& \text { (b) } X\left(\lambda\left[F^{0}(\tilde{w})\right]_{*}+\sum_{j=1}^{d-1} i \eta_{j}\left[F^{j}(\tilde{w})\right]_{*}-\left[A^{d}(\tilde{w}) \tilde{w}^{\prime}\right]_{*}\right)-\left[A^{d}(\tilde{w}) w^{\#}\right]_{*}=0 \text { on } x=0 .
\end{aligned}
$$

Here we've used the relation

$$
\left(A^{d}(\tilde{w}) \tilde{w}^{\prime}\right)^{\prime}=d_{w} R(\tilde{w}) \tilde{w}^{\prime}
$$

obtained by differentiating the profile equation in (2.21). In computing jumps involving the derivative of the profile, $\tilde{w}^{\prime}$, we use

$$
\begin{aligned}
& \lim _{x \rightarrow 0^{+}} \tilde{w}^{\prime}=\tilde{w}_{+}^{\prime}(0)=0 \\
& \lim _{x \rightarrow 0^{-}} \tilde{w}^{\prime}=\tilde{w}_{-}^{\prime}(0)
\end{aligned}
$$

as is appropriate for the linearized transmission problem.

Remark 4.1. The use of a "good unknown" like $w^{\#}=w-X \tilde{w}^{\prime}$ dates back to the paper of Alinhac [Al]. 
In defining the ZND Evans function, $D_{Z N D}$, we'll work with the form of the ZND problem given by (4.7). The limiting systems obtained by letting $x \rightarrow \pm \infty$ in (4.7)(a) are

$$
(Z N D)_{ \pm}\left(\lambda A^{0}\left(w_{ \pm}\right)+\sum_{j=1}^{d-1} i \eta_{j} A^{j}\left(w_{ \pm}\right)\right) \nu+A^{d}\left(w_{ \pm}\right) \partial_{x} \nu=d_{w} R\left(w_{ \pm}\right) \nu
$$

Our analysis will focus mainly on the minus side. With $\nu=(v, \mathrm{y})$ we can write $(4.10)_{-}$as the equivalent $(n+s) \times(n+s)$ system

$$
\partial_{x} \nu=\mathcal{G}\left(w_{-}, \lambda, \eta\right) \nu
$$

where

$$
\begin{aligned}
& \mathcal{G}\left(w_{-}, \lambda, \eta\right)=\left(\begin{array}{cc}
H_{-}(\lambda, \eta) & \left(a^{d}\right)^{-1} Q K \\
0 & -\left(g^{d}\right)^{-1}\left(\lambda g^{0} I_{s}+K\right)
\end{array}\right) \text { with } \\
& H_{ \pm}(\lambda, \eta)=-\left(a^{d}\right)^{-1}\left(\lambda a^{0}+\sum_{j=1}^{d-1} i \eta_{j} a^{j}\right)\left(V_{ \pm}\right) .
\end{aligned}
$$

The matrix $\mathcal{G}\left(w_{+}, \lambda, \eta\right)$ is defined similarly (see (4.25)).

Similarly, we can write the variable-coefficient system (4.7)(a) in the form

$$
\partial_{x} w^{\#}=\mathcal{G}(\tilde{w}(x), \lambda, \eta) w^{\#} \text { in } \pm x>0,
$$

where for some $\delta>0$

$$
\left|\mathcal{G}(\tilde{w}, \lambda, \eta)-\mathcal{G}\left(w_{-}, \lambda, \eta\right)\right| \leq C e^{-\delta|x|} \text { as } x \rightarrow-\infty
$$

uniformly for $|\lambda, \eta|$ in bounded sets.

\subsection{The linearized Chapman-Jouguet system}

The process in this case parallels that for ZND. After the same change of variables (4.1), the CJ problem (2.30), (2.31) takes the form

$$
\begin{aligned}
& \sum_{j=0}^{d-1} \tilde{f}^{j}(V)_{y_{j}}+\tilde{\mathbf{f}}^{d}(V, d X)_{x}=0 \\
& \sum_{j=0}^{d-1} X_{y_{j}}\left[\tilde{f}^{j}(V)\right]-\left[\tilde{f}^{d}(V)\right]=0 \text { on } x=0,
\end{aligned}
$$

where

$$
\tilde{\mathbf{f}}^{d}(V, d X)=\tilde{f}^{d}(V)-\sum_{j=0}^{d-1} X_{y_{j}} \tilde{f}^{j}(V)
$$


Linearizing with respect to both $V$ and $X$ about the stationary solution given by $V_{ \pm}$and the front $X=0$, and taking the Laplace-Fourier transform as before, we get the transmission problem

$$
\begin{aligned}
& \text { (a) } \lambda \tilde{a}^{0} v+\sum_{j=1}^{d-1} i \eta_{j} \tilde{a}^{j} v+\tilde{a}^{d} v^{\prime}=0 \\
& \text { (b) } X\left(\lambda\left[\tilde{f}^{0}\right]+\sum_{j=1}^{d-1} i \eta_{j}\left[\tilde{f}^{j}\right]\right)-\left[\tilde{a}^{d} v\right]=0 \text { on } x=0,
\end{aligned}
$$

where $(v, X)$ now denotes the (transformed) perturbation, $\tilde{a}^{j}:=d \tilde{f}^{j}$, and the $\tilde{f}^{j}, \tilde{a}^{j}$ are evaluated at $V_{ \pm}$in $\pm x>0$. The interior problem (4.17)(a) can be rewritten

$$
\begin{aligned}
& v^{\prime}=\tilde{H}_{ \pm}(\lambda, \eta) v \text { in } \pm x>0, \text { with } \\
& \tilde{H}_{ \pm}(\lambda, \eta)=-\left(\tilde{a}^{d}\right)^{-1}\left(\lambda \tilde{a}^{0}+\sum_{j=1}^{d-1} i \eta_{j} \tilde{a}^{j}\right)\left(V_{ \pm}\right) .
\end{aligned}
$$

Note that (3.5) implies $\tilde{H}_{ \pm}=H_{ \pm}$for $H_{ \pm}$as in (4.12).

\subsection{The Chapman-Jouguet determinant}

Here and below we shall often write functions $f(\lambda, \eta)$ instead as functions $f(\zeta)$ where $\zeta=(\tau, \gamma, \eta)$. First we define the determinant $\Delta_{C J}(\zeta)$, whose zeros in $\mathbb{R}_{+}^{d+1}=\{\zeta: \gamma>0\}$ correspond to solutions of the linearized CJ problem that decay in $x$ but grow exponentially with time.

For $\zeta \in \mathbb{R}_{+}^{d+1}$ let $F_{ \pm}(\zeta)$ be the generalized eigenspace of $H_{ \pm}(\zeta)$ corresponding to eigenvalues with negative (resp. positive) real part. The hyperbolicity hypothesis (H3) implies that the dimensions of $F_{ \pm}(\zeta)$ are constant in $\mathbb{R}_{+}^{d+1}$, so we can set $(\tau, \eta)=0$ in $(4.18)$ and use the assumption of $n$-shock structure (H0) to see that

$$
\operatorname{dim} F_{+}(\zeta)=0 ; \operatorname{dim} F_{-}(\zeta)=n-1 \text { in } \mathbb{R}_{+}^{d+1} .
$$

A classical argument based on conjugation of $H_{-}(\zeta)$ to block structure shows that $F_{-}(\zeta)$ is $C^{\infty}$ in $\mathbb{R}_{+}^{d+1}$ and extends continuously to $\overline{\mathbb{R}}_{+}^{d+1} \backslash 0$ (see $[\mathrm{K}]$ or $[\mathrm{CP}]$, chapter 7 ). So we may choose a basis

$$
s_{-}^{j}(\zeta), j=1, \ldots, n-1
$$

for $F_{-}(\zeta)$, locally near any point $\zeta^{*} \in \overline{\mathbb{R}}_{+}^{d+1} \backslash 0$, where the $s_{-}^{j}$ are homogeneous of degree 0 for $\zeta \neq 0$ and $C^{\infty}$ in $\mathbb{R}_{+}^{d+1}$ with continuous extensions to $\overline{\mathbb{R}}_{+}^{d+1} \backslash 0$ (in fact the $s_{-}^{j}$ extend smoothly away from glancing points). The spaces $F_{-}(\zeta)$ define a vector bundle over the contractible base space $\overline{\mathbb{R}}_{+}^{d+1} \backslash 0$, so in fact the $s_{-}^{j}$ can be chosen globally on $\overline{\mathbb{R}}_{+}^{d+1} \backslash 0$ with the regularity described above. (Contractible base spaces admit only trivial bundles: $[\mathrm{S}]$, 
Cor. 11.6, p. 53). In the case of the physical CJ equations these properties of the $s_{-}^{j}$ can be read off from explicit formulas [E3].

Inspection of (4.17), (4.18) shows that the linearized CJ problem has solutions growing exponentially with time if for some $\zeta \in \mathbb{R}_{+}^{d+1}$ the $n$ vectors

$$
\lambda\left[\tilde{f}^{0}\right]+\sum_{j=1}^{d-1} i \eta_{j}\left[\tilde{f}^{j}\right], \quad r_{-}^{j}(\zeta):=a^{d}\left(V_{-}\right) s_{-}^{j}(\zeta), j=1, \ldots, n-1
$$

are linearly dependent (recall $\tilde{a}^{j}\left(V_{-}\right)=a^{j}\left(V_{-}\right)$).

Introduce polar coordinates $\zeta=\rho \hat{\zeta}$ where $\hat{\zeta} \in S_{+}^{d}=\left\{\zeta \in \mathbb{R}_{+}^{d+1}:|\zeta|=1\right\}$. In view of (3.7) we have

$$
\left[\tilde{f}^{0}\right]=\left[f^{0}\right]+g^{0}\left(V_{+}\right) Q \mathbf{1},\left[\tilde{f}^{j}\right]=\left[f^{j}\right], j=1, \ldots, d-1,
$$

so linear dependence of the vectors (4.21) is equivalent to vanishing of the determinant

$$
\Delta_{C J}(\hat{\zeta})=\operatorname{det}\left(r_{-}^{1}(\hat{\zeta}), \ldots, r_{-}^{n-1}(\hat{\zeta}), \hat{\lambda}\left[f^{0}\right]+\sum_{j=1}^{d-1} i \hat{\eta}_{j}\left[f^{j}\right]+\hat{\lambda} g^{0}\left(V_{+}\right) Q \mathbf{1}\right)
$$

When $Q=0$ this coincides with the Majda determinant for a Lax $n$-shock [Ma1]. Observe that $\Delta_{C J}(\hat{\zeta})$ is $C^{\infty}$ on $S_{+}^{d}$ and has a continuous extension to $\bar{S}_{+}^{d}$.

\subsection{The ZND Evans function $D_{Z N D}(\zeta)$}

Consider the linearized, transformed ZND problem (4.13)

$$
\partial_{x} w^{\#}=\mathcal{G}(\tilde{w}(x), \zeta) w^{\#} \text { in } \pm x>0
$$

together with the jump condition $(4.7)(\mathrm{b})$. In this paper we are exclusively concerned with high frequency behavior, so we shall define $D_{Z N D}(\zeta)=D_{Z N D}(\hat{\zeta}, \rho)$ in $\hat{\gamma}>0, \rho>0$ and show that it has a continuous extension to $\hat{\gamma} \geq 0, \rho>0$. Continuous extension to $\hat{\gamma} \geq 0$, $\rho \geq 0$ is carried out in $[\mathrm{JLW}]$ and requires different arguments.

Let $\zeta^{*}$ denote an arbitrary fixed basepoint in $\mathbb{R}^{d+1}$. The first step in constructing the ZND Evans function is to find, locally near $\zeta^{*}$, a basis for the solutions of (4.24) that decay to zero as $x \rightarrow \pm \infty$. The exponential decay of the ZND profile to its endstates (3.4) will allow us obtain such a basis by considering the limiting problems defined by the matrices

$$
\begin{aligned}
& \mathcal{G}\left(w_{-}, \zeta\right)=\left(\begin{array}{cc}
H_{-}(\zeta) & \left(a^{d}\right)^{-1} Q K \\
0 & -\left(g^{d}\right)^{-1}\left(\lambda g^{0} I_{s}+K\right)
\end{array}\right) \\
& \mathcal{G}\left(w_{+}, \zeta\right)=\left(\begin{array}{cc}
H_{+}(\zeta) & 0 \\
G_{21} & -\left(g^{d}\right)^{-1} \lambda g^{0} I_{s}
\end{array}\right) .
\end{aligned}
$$

We first conjugate the variable coefficient problem (4.24) in $x<0$ to the constant coefficient problem

$$
\nu^{\prime}=\mathcal{G}\left(w_{-}, \zeta\right) \nu \text { in } x<0,
$$

using the following result, whose proof is established in Lemma 2.6 of [MZ1]: 
Lemma 4.2. Fix a basepoint $\zeta^{*} \in \overline{\mathbb{R}}_{+}^{d+1}$ (even $\zeta^{*}=0$ is allowed here), and choose any $\delta^{\prime}$ such that $0<\delta^{\prime}<\delta$, where $\delta$ as in (3.4) governs the exponential decay of $\tilde{w}(x)$. There exists a $C^{\infty},(n+s) \times(n+s)$ matrix $Z_{-}(x, \zeta)$, defined for all $x \leq 0$ and for $\zeta \in \overline{\mathbb{R}}_{+}^{d+1}$ in a neighborhood $\omega$ of $\zeta^{*}$, such that

$$
\begin{aligned}
& \text { (a) } \partial_{x} Z_{-}=\mathcal{G}(\tilde{w}, \zeta) Z_{-}-Z_{-} \mathcal{G}\left(w_{-}, \zeta\right) \text { in } x \leq 0 \\
& \text { (b) }\left|Z_{-}^{-1}\right| \leq C \\
& \text { (c) }\left|\partial_{x}^{k} \partial_{\zeta}^{\alpha}\left(Z_{-}-I\right)\right| \leq C_{k, \alpha} e^{-\delta^{\prime}|x|},
\end{aligned}
$$

for positive constants $C, C_{k, \alpha}$ independent of $x \leq 0, \zeta \in \omega$.

Observe that because of $(4.27)(\mathrm{a}), w^{\#}(x)$ is a solution of (4.24) if and only if $\nu(x)$ defined by

$$
w^{\#}=Z_{-}(x, \zeta) \nu
$$

is a solution of (4.26). The extra properties $(4.27)(\mathrm{b}),(\mathrm{c})$ imply that $Z_{-}$establishes a very useful correspondence between solutions of the two problems.

In $x>0$ we have $\tilde{w}(x)=w_{+}$. It follows directly from (4.19) and $-\left(g^{d}\right)^{-1} g^{0}>0$ that, for $\hat{\gamma}>0, \rho>0$, the only solution of

$$
\nu^{\prime}=\mathcal{G}\left(w_{+}, \zeta\right) \nu \text { in } x>0
$$

decaying to 0 as $x \rightarrow+\infty$ is the trivial solution.

Remark 4.3. In the next section we shall use the fact that a conjugator for the transposed system

$$
\left.\partial_{x} \theta=-\mathcal{G}^{t}(\tilde{w}(x), \zeta)\right) \theta \text { on } x \leq 0
$$

is given by $\left(Z_{-}^{-1}(x, \zeta)\right)^{t}$ for $Z_{-}$as above. In other words, $\theta(x, \zeta)$ is a solution of $(4.30)$ if and only if $\theta_{L}(x, \zeta)$ defined by

$$
\theta(x, \zeta)=\left(Z_{-}^{-1}(x, \zeta)\right)^{t} \theta_{L}(x, \zeta)
$$

is a solution of the limiting problem at $x=-\infty$ :

$$
\partial_{x} \theta_{L}=-\mathcal{G}^{t}\left(w_{-}, \zeta\right) \theta_{L}
$$

This is readily checked using $(4.27)(a)$ and

$$
\partial_{x} Z_{-}^{-1}=-Z_{-}^{-1}\left(\partial_{x} Z_{-}\right) Z_{-}^{-1} .
$$

It is clear from (4.25) that when $\hat{\gamma}>0, \rho>0$ the matrix $\mathcal{G}\left(w_{-}, \zeta\right)$ has no pure imaginary eigenvalues. In fact, from (4.19) and (H2) we see that for $\zeta$ near $\zeta^{*} \in \mathbb{R}_{+}^{d+1}$ there is an $(n-1+s)$-dimensional space of solutions to (4.26) that decay to zero as $x \rightarrow-\infty$. Let $\nu^{j}(x, \zeta), j=1, \ldots, n-1+s$ be a basis for this space and define the initial space

$$
\mathcal{F}_{-}(\zeta):=\operatorname{span}\left\{\nu^{j}(0, \zeta), j=1, \ldots, n-1+s\right\} .
$$


The space

$$
E_{-}(\zeta):=Z_{-}(0, \zeta) \mathcal{F}_{-}(\zeta)
$$

is then the space of initial data of decaying solutions of the variable coefficient problem (4.24) in $x \leq 0$. A basis for $E_{-}(\zeta)$ is given by

$$
w^{j}(0, \zeta), j=1, \ldots, n-1+s, \text { where } w^{j}(x, \zeta):=Z_{-}(x, \zeta) \nu^{j}(x, \zeta) .
$$

In view of the equivalence of the linearized problems (4.7)(a) and (4.13), we see that the linearized transmission problem (4.4) has solutions decaying in $x$ and growing exponentially in time for some $\zeta \in \mathbb{R}_{+}^{d+1}$ when the $n+s$ vectors

$$
A^{d}\left(w^{*}\right) w^{1}(0, \zeta), \ldots, A^{d}\left(w^{*}\right) w^{n-1+s}(0, \zeta), \lambda\left[F^{0}(\tilde{w})\right]_{*}+\sum_{j=1}^{d-1} i \eta_{j}\left[F^{j}(\tilde{w})\right]_{*}+A^{d}\left(w^{*}\right) \tilde{w}^{\prime}\left(0^{-}\right)
$$

are linearly dependent. This is the case if and only if the ZND Evans function

$$
\begin{aligned}
& D_{Z N D}(\zeta)= \\
& \operatorname{det}\left(A^{d}\left(w^{*}\right) w^{1}(0, \zeta), \ldots, A^{d}\left(w^{*}\right) w^{n-1+s}(0, \zeta), \lambda\left[F^{0}(\tilde{w})\right]_{*}+\sum_{j=1}^{d-1} i \eta_{j}\left[F^{j}(\tilde{w})\right]_{*}+A^{d}\left(w^{*}\right) \tilde{w}^{\prime}\left(0^{-}\right)\right)
\end{aligned}
$$

vanishes for such $\zeta$.

The spaces $E_{-}(\zeta)$ define a $C^{\infty}$ vector bundle over $\mathbb{R}_{+}^{d+1}$. Next we show that this bundle extends continuously to $\overline{\mathbb{R}}_{+}^{d+1} \backslash 0=\{\zeta=\rho \hat{\zeta}: \hat{\gamma} \geq 0, \rho>0\}$. The upper triangular structure of $\mathcal{G}\left(w_{-}, \zeta\right)$ is helpful here, but care is needed since eigenvalues of the diagonal blocks may cross.

Proposition 4.4. The bundle $E_{-}(\zeta)$ has a continuous extension from $\mathbb{R}_{+}^{d+1}$ to $\overline{\mathbb{R}}_{+}^{d+1} \backslash 0$.

Proof. 1. No crossing. Let $\zeta^{*} \in \overline{\mathbb{R}}_{+}^{d+1} \backslash 0$ be such that $\gamma^{*}=0$ and suppose that the two diagonal blocks of $\mathcal{G}\left(w_{-}, \zeta^{*}\right)$ have no eigenvalues in common. For $\zeta$ near $\zeta^{*}$ with $\gamma>0$ there is then a smooth decomposition (i.e., with $C^{\infty}$ bounded projections)

$$
E_{-}(\zeta)=E_{H}(\zeta) \oplus E_{K}(\zeta)
$$

where $E_{H}(\zeta)$ (resp., $E_{K}(\zeta)$ ) is the generalized eigenspace of $\mathcal{G}\left(w_{-}, \zeta\right)$ associated to eigenvalues $\mu$ of the upper left block (resp., lower right block) with $\Re \mu>0$. Since $K$ is positive definite, $E_{K}(\zeta)$ has a $C^{\infty}$ extension to $\gamma \geq 0$ for $\zeta$ near $\zeta^{*}$. On the other hand we have

$$
E_{H}(\zeta)=\left\{\left(\begin{array}{l}
u \\
0
\end{array}\right), u \in F_{-}(\zeta)\right\}
$$

for $F_{-}(\zeta)$ as in (4.19), so $E_{H}(\zeta)$ extends continuously to $\gamma \geq 0$. 
2. Crossing. Let $\zeta^{*} \in \overline{\mathbb{R}}_{+}^{d+1} \backslash 0$ be such that $\gamma^{*}=0$. Suppose for the moment that the diagonal blocks of $\mathcal{G}\left(w_{-}, \zeta^{*}\right)$ have a single eigenvalue $\mu_{c}\left(\zeta^{*}\right)$, possibly of high multiplicity, in common. We must have $\Re \mu_{c}>0$ since $K$ is positive definite. For $\zeta$ near $\zeta^{*}$ with $\gamma>0$ we now have a smooth decomposition

$$
E_{-}(\zeta)=E_{H^{\prime}}(\zeta) \oplus E_{K^{\prime}}(\zeta) \oplus E_{c}(\zeta)
$$

where $E_{c}(\zeta)$ is the generalized eigenspace of $\mathcal{G}\left(w_{-}, \zeta\right)$ associated to eigenvalues of $\mathcal{G}\left(w_{-}, \zeta\right)$ near $\mu_{c}\left(\zeta^{*}\right)$, and $E_{H^{\prime}}(\zeta)$ (resp., $E_{K^{\prime}}(\zeta)$ ) is the generalized eigenspace of $\mathcal{G}\left(w_{-}, \zeta\right.$ ) associated to the remaining eigenvalues of the upper left (resp., lower right) block. The second and third summands in (4.40) have $C^{\infty}$ extensions to $\gamma \geq 0$ for $\zeta$ near $\zeta^{*}$, and the first extends continuously to $\gamma \geq 0$ by the same analysis used to treat $F_{-}(\zeta)$.

The case where the diagonal blocks have more than one eigenvalue in common is handled in essentially the same way.

We now have the bundle $E_{-}(\zeta)$ continuously extended to $\overline{\mathbb{R}}_{+}^{d+1} \backslash 0$. Using contractibility of the base space and Gram-Schmidt, we obtain

Corollary 4.5. The functions $w^{j}(0, \zeta), j=1, \ldots, n-1+s$ appearing in (4.37) can be chosen orthonormal and to be $C^{\infty}$ in $\mathbb{R}_{+}^{d+1}$ with continuous extensions to $\overline{\mathbb{R}}_{+}^{d+1} \backslash 0$. Thus, $D_{Z N D}(\zeta)$ as in (4.37) is globally defined on $\overline{\mathbb{R}}_{+}^{d+1} \backslash 0$ and has the same regularity as the $w^{j}(0, \zeta)$.

Remark 4.6. In the case of the physical ZND equations one can see using the explicit formulas for eigenvalues (see (6.3)) that crossing of eigenvalues associated to different blocks does not occur; thus only part 1. of the above proof is needed for the physical equations. However, crossing of real parts always occurs.

\subsection{Relation to Erpenbeck's stability function $V(\zeta)$.}

In this section we define another ZND Evans function, $\mathbb{D}_{Z N D}(\zeta)$, which turns out to be just a nonvanishing multiple of $D_{Z N D}(\zeta)$. The new Evans function is more convenient for computation and for analysis of the high frequency limit. In addition we show

$$
\mathbb{D}_{Z N D}(\zeta)=V(\zeta)
$$

where $V(\zeta)$ is the stability function defined by Erpenbeck in [E1]. Erpenbeck worked directly with the inhomogeneous interior equation for $w$ in (4.5), instead of using the new unknown $w^{\#}$ (4.6) as we have done to reduce to a homogeneous problem (4.7).

For $\zeta \in \mathbb{R}_{+}^{d+1}$ let $\Phi(x, \zeta)$ be a fundamental matrix for (4.24) on $x \leq 0$. Thus, $\Phi(x, \zeta)$ is an $(n+s) \times(n+s)$ matrix whose columns

$$
w^{j}(x, \zeta), j=1, \ldots, n+s
$$

are linearly independent solutions of (4.24) on $x \leq 0$. Here we suppose that for $j=$ $1, \ldots, n-1+s$ the initial values $w^{j}(0, \zeta)$ are given by the functions chosen in Corollary 4.5. 
Thus, $w^{n+s}(x, \zeta)$ grows exponentially while the other columns of $\Phi$ decay exponentially to zero as $x \rightarrow-\infty$.

It is easily checked that $\left(\Phi^{-1}\right)^{t}$ is then a fundamental matrix for the system

$$
\partial_{x} \theta=-\mathcal{G}^{t}(\tilde{w}(x), \zeta) \theta \text { on } x \leq 0 .
$$

Let $\theta^{n+s}$ be the last row of $\Phi^{-1}$ and note that because $\Phi^{-1} \Phi=I$ we have

$$
\theta^{n+s}(x, \zeta) \cdot w^{j}(x, \zeta)=0, j=1, \ldots, n+s-1 .
$$

In other words $\bar{\theta}^{n+s}(x, \zeta)$ is orthogonal to the $w^{j}(x, \zeta)$ in $\mathbb{C}^{n+s}$, where the overline denotes complex conjugate.

The norm of $\Phi^{-1}$ is generally unbounded as $\gamma \downarrow 0$, so it is not clear that $\theta^{n+s}(0, \zeta)$ has a continuous extension to $\overline{\mathbb{R}}^{d+1} \backslash 0$. To arrange this note that by Remark 4.3, a nonvanishing multiple $c(\zeta) \theta^{n+s}(0, \zeta)$ can be constructed as

$$
\left(Z_{-}^{-1}(0, \zeta)\right)^{t} f_{+}(\zeta)
$$

where $Z_{-}$is as in (4.28) and $f_{+}(\zeta) \in C^{\infty}\left(\mathbb{R}_{+}^{d+1}\right)$ is an eigenvector of the limit matrix $-\mathcal{G}^{t}\left(w_{-}, \zeta\right)$ associated to the unique eigenvalue $\mu_{+}(\zeta)$ with positive real part. Since $f_{+}(\zeta)$ can be continuously extended to $\overline{\mathbb{R}}_{+}^{d+1} \backslash 0$, this shows that

$$
B(\zeta):=\operatorname{span}\left\{\theta^{n+s}(0, \zeta)\right\}
$$

is a smooth line bundle on $\mathbb{R}_{+}^{d+1}$ with a continuous extension to $\overline{\mathbb{R}}_{+}^{d+1} \backslash 0$. Now redefine $\theta^{n+s}(0, \zeta)$ to be a globally defined basis vector for $B(\zeta)$ with the same regularity, and set

$$
\tilde{\theta}(\zeta):=\frac{\theta^{n+s}(0, \zeta)}{\left|\theta^{n+s}(0, \zeta)\right|} \text { for } \zeta \in \overline{\mathbb{R}}_{+}^{d+1} \backslash 0 .
$$

Definition 4.7. Denote

$$
J(\zeta)=\lambda\left[F^{0}(\tilde{w})\right]_{*}+\sum_{j=1}^{d-1} i \eta_{j}\left[F^{j}(\tilde{w})\right]_{*},
$$

and set

$$
\mathbb{D}_{Z N D}(\zeta):=\tilde{\theta}(\zeta) \cdot\left(\left(A^{d}\left(w^{*}\right)\right)^{-1} J(\zeta)+\tilde{w}^{\prime}\left(0^{-}\right)\right)
$$

Proposition 4.8. For $\zeta \in \overline{\mathbb{R}}_{+}^{d+1} \backslash 0$ we have, up to a sign,

$$
D_{Z N D}(\zeta)=\operatorname{det} A^{d}\left(w^{*}\right) \mathbb{D}_{Z N D}(\zeta) .
$$

Proof. This follows directly from the definition of $D_{Z N D}(\zeta)$ after expressing

$$
\left(A^{d}\left(w^{*}\right)\right)^{-1} J(\zeta)+\tilde{w}^{\prime}\left(0^{-}\right)
$$

as a linear combination of the orthonormal functions

$$
\overline{\tilde{\theta}}(\zeta) \text { and } w^{j}(0, \zeta), j=1, \ldots, n-1+s .
$$


In order to relate $\mathbb{D}_{Z N D}(\zeta)$ to Erpenbeck's stability function $V(\zeta)$ [E1], we use the following result proved in the appendix of [E1].

Proposition 4.9. Let $\tilde{\theta}(x, \zeta)$ be the solution of (4.43) with initial data $\tilde{\theta}(\zeta)$ as in (4.47). Fix $\zeta$ with $\gamma>0$ and consider the inhomogeneous problem

$$
\phi^{\prime}(x)=\mathcal{G}(\tilde{w}(x), \zeta) \phi+f(x) \text { on } x \leq 0,
$$

for $f(x)$ continuous and bounded on $x \leq 0$. Then a solution $\phi$ is bounded on $x \leq 0$ if and only if

$$
\tilde{\theta}(0) \cdot \phi(0)=\int_{-\infty}^{0} \tilde{\theta}(s, \zeta) \cdot f(s) d s
$$

From (4.8) and (4.5) we see that for any $X, X \tilde{w}^{\prime}(x)$ is a bounded solution of (4.50) with

$$
f(x)=X\left(A^{d}(\tilde{w})\right)^{-1}\left(\lambda A^{0}(\tilde{w}) \tilde{w}^{\prime}+\sum_{j=1}^{d-1} i \eta_{j} A^{j}(\tilde{w}) \tilde{w}^{\prime}\right):=K(x, X, \zeta) .
$$

Taking $X=1$ and applying Proposition 4.9 we find

$$
\tilde{\theta}(0) \cdot \tilde{w}^{\prime}(0)=\int_{-\infty}^{0} \tilde{\theta}(s, \zeta) \cdot K(s, 1, \zeta) d s .
$$

We conclude for $\zeta \in \mathbb{R}_{+}^{d+1}$ (recall Definition 4.7)

$$
\mathbb{D}_{Z N D}(\zeta)=\tilde{\theta}(\zeta) \cdot\left(A^{d}\left(w^{*}\right)\right)^{-1} J(\zeta)+\int_{-\infty}^{0} \tilde{\theta}(s, \zeta) \cdot K(s, 1, \zeta) d s:=V(\zeta),
$$

where $V(\zeta)$ is the stability function defined in [E1], equation (4.11). The terms involving $b_{1}$ and $b_{2}$ there correspond to the integral term in (4.54).

Since $V(\zeta)$ extends continuously to $\overline{\mathbb{R}}_{+}^{d+1} \backslash 0$ and agrees with $\mathbb{D}_{Z N D}$ on $\mathbb{R}_{+}^{d+1}$, we have proved

Proposition 4.10. $D_{Z N D}(\zeta)=\operatorname{det} A^{d}\left(w^{*}\right) \mathbb{D}_{Z N D}(\zeta)=\operatorname{det} A^{d}\left(w^{*}\right) V(\zeta)$ on $\overline{\mathbb{R}}_{+}^{d+1} \backslash 0$.

\subsection{Definition of $\Delta_{Z N D}$}

Here we define $\Delta_{Z N D}$, an analogue of Majda's determinant for the von Neumann shock. This turns out to be the governing determinant for the problem of finite time existence of slightly curved ZND fronts. In the next section we relate $\Delta_{Z N D}$ to the high frequency limit of $\mathbb{D}_{Z N D}(\hat{\zeta}, \rho) / \rho$.

Consider again the nonlinear ZND system (4.2), but now with the reaction term $R(w)$ set equal to zero. Linearizing with respect to both $w$ and $X$ around the planar front $X=0$ and the von Neumann state

$$
w_{v N}:=\left\{\begin{array}{l}
w^{*}, x<0 \\
w_{+}, x>0
\end{array}\right.
$$


we obtain

$$
\begin{aligned}
& \sum_{j=0}^{d-1} A^{j}\left(w_{v N}\right) \partial_{j} w+A^{d}\left(w_{v N}\right) \partial_{x} w=0 \text { in } \pm x \geq 0 \\
& \sum_{j=0}^{d-1} \partial_{j} \phi\left[F^{j}\left(w_{v N}\right)\right]-\left[A^{d}\left(w_{v N}\right) w\right]=0 \text { on } x=0 .
\end{aligned}
$$

Next, we Fourier-Laplace transform (4.56) in $\left(t, y^{\prime}\right)$ to find (suppressing hats on $\phi$ and $w$ ):

$$
\begin{aligned}
& w^{\prime}=\mathcal{G}_{1}\left(w_{v N}, \zeta\right) w:=-\left(A^{d}\left(w_{v N}\right)\right)^{-1}\left(\lambda A^{0}\left(w_{v N}\right)+\sum_{j=1}^{d-1} i \eta_{j} A^{j}\left(w_{v N}\right)\right) w \text { in } \pm x \geq 0 \\
& \phi\left(\lambda\left[F^{0}\left(w_{v N}\right)\right]+\sum_{j=1}^{d-1} i \eta_{j}\left[F^{j}\left(w_{v N}\right)\right]\right)-\left[A^{d}\left(w_{v N}\right) w\right]=0 \text { on } x=0 .
\end{aligned}
$$

We may write

$$
\mathcal{G}_{1}\left(w_{v N}, \zeta\right)=\left(\begin{array}{cc}
H\left(w_{v N}, \zeta\right) & 0 \\
g_{21}\left(w_{v N}, \zeta\right) & -\left(g^{d}\right)^{-1} \lambda g^{0} I_{s}
\end{array}\right)
$$

The hyperbolicity assumption (H3) implies that for $\zeta \in \mathbb{R}_{+}^{d+1}$ the eigenvalues of $\mathcal{G}_{1}\left(w_{v N}, \zeta\right)$ have nonzero real parts. We conclude from assumptions (H0) and (H1) (recall Remark 3.2) that there are $n-1+s$ such eigenvalues for the left state $w^{*}$ and $n+s$ for the right state $w_{+}$. Thus, the decaying eigenspace for $\mathcal{G}_{1}\left(w_{+}, \zeta\right)$ is trivial. Let

$$
S_{-}^{j}(\zeta), j=1, \ldots, n-1+s
$$

be a $C^{\infty}$ basis, homogeneous of degree 0 , for the decaying generalized eigenspace of $\mathcal{G}_{1}\left(w^{*}, \zeta\right)$ and set $R_{-}^{j}=A^{d}\left(w^{*}\right) S_{-}^{j}$. Observe that the problem (4.56) has solutions decaying in $x$ but exponentially growing in $t$ if the following $(n+s) \times(n+s)$ determinant vanishes:

$$
\Delta_{Z N D}(\hat{\zeta})=\operatorname{det}\left(R_{-}^{1}(\hat{\zeta}), \ldots, R_{-}^{n-1+s}(\hat{\zeta}), \hat{\lambda}\left[F^{0}\left(w_{v N}\right)\right]+\sum_{j=1}^{d-1} i \hat{\eta}_{j}\left[F^{j}\left(w_{v N}\right)\right]\right) .
$$

Hypothesis (H3) allows us to apply the result of [Met2] to obtain continuous extensions of the $S_{-}^{j}(\hat{\zeta})$, and thus of $\Delta_{Z N D}(\hat{\zeta})$, to $\bar{S}_{+}^{d}$.

Finally, let us rewrite $\Delta_{Z N D}(\hat{\zeta})$ in a form similar to (4.48). For $\hat{\gamma}>0$ observe that the $S_{-}^{j}(\hat{\zeta})$ can be chosen orthonormal. Let $\theta^{*}(\hat{\zeta}) \in C^{\infty}\left(S_{+}^{d}\right)$ be a unit left eigenvector of $\mathcal{G}_{1}\left(w^{*}, \hat{\zeta}\right)$ associated to the unique eigenvalue with negative real part. We have then

$$
\theta^{*}(\hat{\zeta}) \cdot S_{-}^{j}(\hat{\zeta})=0, j=1, \ldots, n-1+s .
$$

As in the proof of Proposition 4.8 we obtain immediately

$$
\Delta_{Z N D}(\hat{\zeta})=\operatorname{det} A^{d}\left(w^{*}\right) \theta^{*}(\hat{\zeta}) \cdot\left(A^{d}\left(w^{*}\right)\right)^{-1}\left(\hat{\lambda}\left[F^{0}\left(w_{v N}\right)\right]+\sum_{j=1}^{d-1} i \hat{\eta}_{j}\left[F^{j}\left(w_{v N}\right)\right]\right) .
$$

Since $\theta^{*}$ extends continuously to $\bar{S}_{+}^{d}$, the equality (4.62) holds on $\bar{S}_{+}^{d}$. 


\section{The high frequency regime.}

The first goal of this section is to prove the following Theorem:

Theorem 5.1. (a) For $\hat{\gamma}>0$ we have up to a sign

$$
\lim _{\rho \rightarrow \infty} \frac{D_{Z N D}(\hat{\zeta}, \rho)}{\rho}=\Delta_{Z N D}(\hat{\zeta})
$$

For any fixed $c>0$, the limit is uniform for $\hat{\zeta}$ satisfying $\hat{\gamma} \geq c$.

(b) For the physical ZND equations, in the case when $\hat{\gamma}=0$ the limit (5.1) holds uniformly for $\hat{\zeta}$ satisfying

$$
|\hat{\lambda}| \leq|\hat{\eta}|\left(\min _{x \leq 0} \sqrt{c^{2}-u_{d}^{2}}\right)-\epsilon_{0}
$$

for any fixed $\epsilon_{0}>0$. Here $c=c(x)$ is the sound speed and $u_{d}(x)$ is the (subsonic) gas speed in the steady reaction zone.

\subsection{Tracking Lemma and high frequency limit of $D_{Z N D}(\zeta) /|\zeta|$.}

We will prove Theorem 5.1 using the following general result, the Tracking Lemma, for ODEs with slowly varying coefficients.

Lemma 5.2 ([Z1],Cor. 8.25;[MaZ]). Consider the $N \times N$ system with $C^{1}$ coefficients

$$
w^{\prime}=(A(x, \delta)+\Theta(x, \delta)) w \text { on } x \leq 0
$$

where for some $C>0$

$$
\left|\partial_{x} A\right|+|\Theta| \leq C \delta,|A| \leq C \text { for } x \leq 0, \delta \in[0,1] .
$$

Suppose also that for some constants $\mu_{*}, \mu^{*}$, the eigenvalues of $A(x, \delta)$ divide into two groups:

$$
\Re \mu_{j}(x, \delta) \leq \mu_{*} \leq 0<\mu^{*} \leq \Re \mu_{k}(x, \delta) \text { for all }(x, \delta),
$$

where $j \leq l, k \geq l+1$ for some $l$. Set $\eta:=\mu^{*}-\mu_{*}$ and let $\mathcal{N}(x, \delta)$ and $\mathcal{P}(x, \delta)$ be the spectral projections onto the generalized eigenspaces of $A(x, \delta)$ associated to $\left\{\mu_{j}, j \leq l\right\}$ and $\left\{\mu_{k}, k \geq l+1\right\}$ respectively. Then there exists a constant $C^{*}$ depending only on $C$ in (5.4) such that solutions $w$ of (5.3) decaying exponentially to 0 as $x \rightarrow-\infty$ satisfy

$$
\frac{|\mathcal{N}(x, \delta) w(x)|}{|\mathcal{P}(x, \delta) w(x)|} \leq \frac{C^{*} \delta}{\eta} \text { for } \delta \leq \frac{\eta}{C^{*}}, x \leq 0 .
$$

Remark 5.3. The lemma shows that for $\delta$ small enough, decaying solutions $w$ of the problem (5.3) with slowly varying coefficients have the property that for each $x, w(x)$ always lie close to, or approximately tracks, the positive eigenspace of the matrix $A(x, \delta)$ frozen at $x$. 
Proof of Theorem 5.1. 1. For $\hat{\gamma}>0$ let $\tilde{\theta}(x, \zeta)$ be the solution to

$$
\partial_{x} \tilde{\theta}=-\mathcal{G}^{t}(\tilde{w}(x), \zeta) \tilde{\theta} \text { on } x \leq 0, \tilde{\theta}(0, \zeta)=\tilde{\theta}(\zeta)
$$

for $\tilde{\theta}(\zeta)$ as in (4.47). We may write

$$
\mathcal{G}(\tilde{w}(x), \zeta)=\mathcal{G}_{1}(\tilde{w}(x), \zeta)+\mathcal{G}_{0}(\tilde{w}(x)),
$$

where $\mathcal{G}_{1}$ is homogeneous of order one in $\zeta$. For $y \leq 0$ set $\psi(y, \hat{\zeta}, \rho):=\tilde{\theta}\left(\frac{y}{\rho}, \zeta\right)$, so $\psi$ satisfies

$$
\partial_{y} \psi=-\mathcal{G}_{1}^{t}\left(\tilde{w}\left(\frac{y}{\rho}\right), \hat{\zeta}\right) \psi-\frac{1}{\rho} \mathcal{G}_{0}^{t}\left(\tilde{w}\left(\frac{y}{\rho}\right)\right) \psi, \quad \psi(0, \hat{\zeta}, \rho)=\tilde{\theta}(\zeta) .
$$

2. From (4.48) we have

$$
\frac{\mathbb{D}_{Z N D}(\hat{\zeta}, \rho)}{\rho}=\tilde{\theta}(\zeta) \cdot\left(\left(A^{d}\left(w_{*}\right)\right)^{-1} J(\hat{\zeta})+\frac{\tilde{w}^{\prime}\left(0^{-}\right)}{\rho}\right) .
$$

Thus, in view of (4.62) to prove (5.1) it suffices to show

$$
\lim _{\rho \rightarrow \infty} \psi(0, \hat{\zeta}, \rho)=\theta^{*}(\hat{\zeta})
$$

up to a sign.

3. Since $\theta^{*}(\hat{\zeta})$ is a left eigenvector of $\mathcal{G}_{1}\left(w^{*}, \hat{\zeta}\right)$ associated to the unique eigenvalue with negative real part, it is a right eigenvector of $-\mathcal{G}_{1}^{t}\left(w^{*}, \hat{\zeta}\right)$ associated to the unique eigenvalue with positive real part. The limit (5.11) now follows by applying Lemma 5.2 to the $(n+s) \times(n+s)$ system (5.9) with the small parameter $\delta=\frac{1}{\rho}$. Observe that

$$
A(y, \delta):=-\mathcal{G}_{1}^{t}\left(\tilde{w}\left(\frac{y}{\rho}\right), \hat{\zeta}\right)
$$

satisfies (5.4) for some $C>0$ uniformly with respect to $\hat{\zeta}$. Assumptions (H0), (H1), and (H3) together with compactness of the orbit $\{\tilde{w}(x),-\infty \leq x \leq 0\}$ imply that (5.5) is satisfied with

$$
\eta=\mu^{*}-\mu_{*} \geq \epsilon_{0} \hat{\gamma} \text { for some } \epsilon_{0}>0
$$

and with the single eigenvalue $\mu_{k}=\mu_{n+s}$ on the far right. Applying (5.6) at $y=0$ yields

$$
\frac{\left|\mathcal{N}\left(0, \frac{1}{\rho}\right) \psi(0, \hat{\zeta}, \rho)\right|}{\left|\mathcal{P}\left(0, \frac{1}{\rho}\right) \psi(0, \hat{\zeta}, \rho)\right|} \leq \frac{C^{*}}{\epsilon_{0} \rho \hat{\gamma}} \text { for } \rho \geq \frac{C^{*}}{\epsilon_{0} \hat{\gamma}} .
$$

Recalling that $\psi(0, \hat{\zeta}, \rho)$ and $\theta^{*}(\hat{\zeta})$ are both unit vectors and that $\theta^{*}(\hat{\zeta})$ spans the positive eigenspace of $-\mathcal{G}_{1}^{t}\left(w^{*}, \hat{\zeta}\right)$, we deduce (5.11) with uniform convergence for $\hat{\gamma} \geq c$.

4. The eigenvalues of $-\mathcal{G}_{1}^{t}(\tilde{w}(x), \hat{\zeta})$ in the case of the physical ZND equations (see (6.3)) are the $(d+s)$-fold $\hat{\lambda} / u_{d}$ together with

$$
\beta_{ \pm}(x, \hat{\zeta})=\frac{\hat{\lambda} u_{d} \pm c \sqrt{\hat{\lambda}^{2}+\left(c^{2}-u_{d}^{2}\right)\left|\hat{\eta}^{2}\right|}}{u_{d}^{2}-c^{2}} .
$$


So for $\hat{\zeta}$ satisfying $\hat{\gamma}=0$ and (5.2), we have

$$
\Re \beta_{-}(x, \hat{\zeta}) \geq C_{0} \sqrt{\epsilon_{0}},
$$

where $C_{0}$ depends just on the profile and is independent of $x$ and $\hat{\zeta}$, while the other eigenvalues all have real part $\leq 0$. Thus, the result follows by applying Lemma 5.2 as before with $\delta=1 / \rho$ and $\eta=\mu^{*}-\mu_{*} \geq C_{0} \sqrt{\epsilon_{0}}$.

\subsection{Asymptotic behavior as both $x \rightarrow-\infty$ and $|\zeta| \rightarrow \infty$.}

Consider again the solution $\tilde{\theta}(x, \zeta)$ to

$$
\partial_{x} \tilde{\theta}=-\mathcal{G}^{t}(\tilde{w}(x), \zeta) \tilde{\theta}, \quad \tilde{\theta}(0, \zeta)=\tilde{\theta}(\zeta) \text { on } x \leq 0
$$

for $\tilde{\theta}(\zeta)$ as in (4.47), and the corresponding limiting problem at $x=-\infty$ :

$$
\partial_{x} \theta=-\mathcal{G}^{t}\left(w_{-}, \zeta\right) \theta \text { on } x \leq 0 .
$$

We know that for $|x|$ large enough and $\zeta$ fixed, $\tilde{\theta}(x, \zeta)$ will be "close" to a decaying solution $\theta_{L}(x, \zeta)$ of (5.17). In this section we consider the question of how the size of $|x|$ must grow with $|\zeta|$ in order for $\tilde{\theta}(x, \zeta)$ and $\theta_{L}(x, \zeta)$ to be close for $|\zeta|$ large. As explained in the Introduction, this information is needed to complete an argument in the proof of the Instability Theorem of [E3].

The properties of the conjugator

$$
\mathcal{Z}(x, \zeta):=\left(Z_{-}^{-1}(x, \zeta)\right)^{t}(\text { Lemma } 4.2 \text { and Remark }(4.3))
$$

imply that for $\zeta$ in a neighborhood $\omega$ of a fixed basepoint $\zeta^{*} \in \overline{\mathbb{R}}_{+}^{d+1}$, the solution $\tilde{\theta}$ is close to a solution $\theta_{L}$ of the limiting problem (5.17) for $|x|$ sufficiently large. More precisely, letting $\mu_{+}(\zeta)$ denote the unique eigenvalue of $-\mathcal{G}^{t}\left(w_{-}, \zeta\right)$ with positive real part when $\gamma>0$, we have

$$
\left|\tilde{\theta}(x, \zeta)-\theta_{L}(x, \zeta)\right| e^{-\mu_{+}(\zeta) x} \leq C(\zeta) e^{-\delta^{\prime}|x|} \text { for } x \leq 0, \zeta \in \omega, \text { and } 0<\delta^{\prime}<\delta
$$

for $\delta$ as in (3.4), where $C(\zeta)$ is uniformly bounded for $\zeta \in \omega$. This follows immediately from

$$
|\mathcal{Z}(x, \zeta)-I| \leq C(\zeta) e^{-\delta^{\prime}|x|} \text { for } \zeta \in \omega
$$

and the fact that $\theta_{L}:=\mathcal{Z}^{-1} \tilde{\theta}$ is given by

$$
\theta_{L}(x, \zeta)=e^{\mu_{+}(\zeta) x} g_{+}(\zeta), \quad \zeta \in \omega
$$

for $g_{+}(\zeta)$ an eigenvector of $-\mathcal{G}^{t}\left(w_{-}, \zeta\right)$ associated to $\mu_{+}(\zeta)$.

Remark 5.4. Since the conjugator $\mathcal{Z}(x, \zeta)$ is only defined locally near fixed basepoints $\zeta^{*}$, we cannot use (5.19) to answer the question about the growth of $|x|$ posed above. Moreover, the proof of the conjugation lemma, Lemma 4.2, gives no information on how fast $C(\zeta)$ in (5.19) grows as $|\zeta| \rightarrow \infty$. 
The first step is to define $\theta_{L}(x, \zeta)$ smoothly and globally in $\zeta$ :

Lemma 5.5. The limit

$$
\lim _{x \rightarrow-\infty} \tilde{\theta}(x, \zeta) e^{-\mu_{+}(\zeta) x}:=g_{+}(\zeta)
$$

exists for all $\zeta \in \overline{\mathbb{R}}_{+}^{d+1} \backslash 0$ and defines a function $g_{+}(\zeta)$ that is $C^{\infty}$ in $\mathbb{R}_{+}^{d+1}$ with a continuous extension to $\overline{\mathbb{R}}_{+}^{d+1} \backslash 0$. For each $\zeta, g_{+}(\zeta)$ is an eigenvector of $-\mathcal{G}^{t}\left(w_{-}, \zeta\right)$ associated to the eigenvalue $\mu_{+}(\zeta)$. Thus, we can define

$$
\theta_{L}(x, \zeta):=e^{\mu_{+}(\zeta) x} g_{+}(\zeta) \text { for } x \leq 0, \zeta \in \overline{\mathbb{R}}_{+}^{d+1} \backslash 0
$$

and $\theta_{L}(x, \zeta)$ is $C^{\infty}$ in $x$ with the same regularity in $\zeta$ as $g_{+}(\zeta)$.

Proof. Note that $\tilde{\theta}(x, \zeta)$ and $\mu_{+}(\zeta)$, which is a simple eigenvalue for $\gamma>0$, are globally defined with the same regularity in $(x, \zeta)$ as claimed for $\theta_{L}$. Fix a basepoint $\zeta^{*}$ and note that for $\zeta$ in a neighborhood $\omega$ of $\zeta^{*}, \mathcal{Z}^{-1} \tilde{\theta}$ is a decaying solution of the limiting problem (5.17) with the stated regularity, and so must have the form

$$
\mathcal{Z}^{-1} \tilde{\theta}=e^{\mu_{+}(\zeta) x} g_{+}(\zeta), \zeta \in \omega
$$

for an eigenvector $g_{+}$associated to $\mu_{+}$. In view of the known properties of $\tilde{\theta}, \mu_{+}$, and $\mathcal{Z}$, (5.24) implies both that $g_{+}$has the claimed regularity in $\omega$ and that the limit (5.22) holds for $\zeta \in \omega$. Uniqueness of the limit then implies that the various locally defined functions $g_{+}$agree on overlapping neighborhoods $\omega$.

We can now make sense of the following definition:

Definition 5.6. For any $\epsilon>0$ and $\zeta \in \overline{\mathbb{R}}_{+}^{d+1} \backslash 0$ let $M^{*}(\epsilon, \zeta)$ be the infimum of the set of $M>0$ such that

$$
\left|\tilde{\theta}(x, \zeta)-\theta_{L}(x, \zeta)\right| e^{-\mu_{+}(\zeta) x} \leq \epsilon \quad \text { for } x \leq-M .
$$

It is sometimes important to know how fast $M^{*}(\epsilon, \zeta)$ grows with $|\zeta|$; for example, the proof of the Instability Theorem of [E3] implicitly relies on an estimate of the growth of $M^{*}(\epsilon, \zeta)$. Such an estimate was not given in [E3], but we give one here that suffices to complete the argument of [E3], section III. Since $\mu_{+}(\zeta)$ is an extreme eigenvalue, we can modify the proof of the Gap Lemma in [Z1] to give a simple proof of the following Proposition. Recall from Assumption 3.1 that

$$
\left|\tilde{w}(x)-w_{-}\right| \leq C e^{-\delta|x|} \text { for } x \leq 0 .
$$

Proposition 5.7. (a) Let $\delta$ be as in (5.26) and set $\zeta=\rho \hat{\zeta}$. There exist positive constants $C_{1}, C_{2}$ independent of $\hat{\zeta} \in \bar{S}_{+}^{d}, \rho \geq 2$ such that

$$
\left|\tilde{\theta}(x, \zeta)-\theta_{L}(x, \zeta)\right| e^{-\mu_{+}(\zeta) x} \leq C_{1} \rho^{n+s+1} e^{-\delta|x|} \text { for } x \leq-C_{2} \ln \rho .
$$

(b)For $\hat{\zeta} \in \bar{S}_{+}^{d}, \rho \geq 2$, and $\epsilon>0$ let $M^{*}(\epsilon, \zeta)$ be as in Definition 5.6. We have

$$
M^{*}(\epsilon, \zeta) \leq \max \left\{C_{2} \ln \rho, \frac{\ln \frac{C_{1}}{\epsilon}+(n+s+1) \ln \rho}{\delta}\right\} .
$$


The main point of part (b) is that $M^{*}(\epsilon, \zeta)$ grows no faster than $\ln \rho$ as $\rho \rightarrow \infty$.

Proof. 1. Preliminaries. Since for $\gamma>0$ the positive eigenspace of $-\mathcal{G}^{t}\left(w_{-}, \zeta\right)$ is one dimensional, the solution $\tilde{\theta}$ of $(5.16)$ is also uniquely characterized by the requirements that it satisfy

$$
\partial_{x} \tilde{\theta}=-\mathcal{G}^{t}(\tilde{w}(x), \zeta) \tilde{\theta} \text { on } x \leq 0
$$

and the asymptotic condition (5.22). We use this observation to construct $\tilde{\theta}$ by a fixed point argument.

In this proof " $C$ " is always a positive constant independent of $(x, \zeta)$ that may change from term to term.

2. Set

$$
g(x, \zeta):=\tilde{\theta}(x, \zeta) e^{-\mu_{+}(\zeta) x}
$$

and note that $\tilde{\theta}$ satisfies (5.29) if and only if $g(x, \zeta)$ satisfies

$$
\partial_{x} g=\left(-\mathcal{G}^{t}\left(w_{-}, \zeta\right)-\mu_{+}(\zeta)\right) g+E(x, \zeta) g,
$$

where for $E(x, \zeta):=-\mathcal{G}^{t}(\tilde{w}(x), \zeta)+\mathcal{G}^{t}\left(w_{-}, \zeta\right)$ we have the estimate

$$
|E(x, \zeta)| \leq C \rho e^{-\delta|x|} .
$$

Set $A_{-}(\zeta):=-\mathcal{G}^{t}\left(w_{-}, \zeta\right)$. Treating $\zeta$ as a parameter, we'll construct $g(x, \zeta)$ satisfying $(5.31)$ as a fixed point of the map

$$
\mathcal{T} g(x, \zeta):=g_{+}(\zeta)+\int_{-\infty}^{x} e^{\left(A_{-}(\zeta)-\mu_{+}(\zeta)\right)(x-y)} E(y, \zeta) g(y, \zeta) d y
$$

on $L^{\infty}(-\infty,-M]$ for $M=M(\rho)$ large enough.

3. Choose $\delta^{\prime}$ such that $0<\delta^{\prime}<\delta$. We claim

$$
\left|e^{\left(A_{-}(\zeta)-\mu_{+}(\zeta)\right) r}\right| \leq C \rho^{n+s} e^{\delta^{\prime} r} \text { for } r \geq 0 .
$$

To prove this we use Dunford's integral [Ka] to write

$$
e^{\left(A_{-}(\zeta)-\mu_{+}(\zeta)\right) r}=\frac{1}{2 \pi i} \int_{\Gamma(\zeta)} e^{\left(z-\mu_{+}(\zeta)\right) r} R(\zeta, z) d z,
$$

where $\Gamma(\zeta)$ is a contour enclosing all the eigenvalues $\mu_{j}(\zeta)$ of $A_{-}(\zeta)$ and $R(\zeta, z):=(z-$ $\left.A_{-}(\zeta)\right)^{-1}$ is the resolvent. Since

$$
\Re \mu_{j}(\zeta)-\Re \mu_{+}(\zeta) \leq 0 \text { for all } j,
$$

we can choose the contours to have length $|\Gamma(\zeta)| \leq C|\zeta|$ and to be such that for all $z \in \Gamma(\zeta)$ we have:

$$
\begin{aligned}
& \Re\left(z-\mu_{+}(\zeta)\right)<\delta^{\prime} \\
& \left|z-\mu_{j}(\zeta)\right| \geq \frac{\delta^{\prime}}{2} \text { for all } j .
\end{aligned}
$$


The estimate (5.34) now follows directly from (5.35), (5.37), and the resolvent estimate

$$
|R(\zeta, z)| \leq C \frac{\left(\left|A_{-}(\zeta)\right|+|z|\right)^{n+s-1}}{\prod_{j=1}^{n+s}\left|z-\mu_{j}(\zeta)\right|} \leq C \rho^{n+s-1} \text { for } z \in \Gamma(\zeta)
$$

4. Applying (5.32) and (5.34) we find for some $C>0$

$$
\begin{aligned}
& \left|\left(\mathcal{T} g_{1}-\mathcal{T} g_{2}\right)(x, \zeta)\right| \leq C\left|g_{1}-g_{2}\right|_{L^{\infty}(-\infty,-M]} \rho^{n+s+1} \int_{-\infty}^{x} e^{\delta^{\prime}(x-y)} e^{\delta y} d y \\
& =\left.C\left|g_{1}-g_{2}\right|_{L^{\infty}(-\infty,-M]} \rho^{n+s+1} e^{\delta^{\prime} x} e^{\left(\delta-\delta^{\prime}\right) y \mid}\right|_{-\infty} ^{x}=C\left|g_{1}-g_{2}\right|_{L_{(-\infty,-M]}^{\infty}} \rho^{n+s+1} e^{\delta x} \\
& \leq C\left|g_{1}-g_{2}\right|_{L^{\infty}(-\infty,-M]} \rho^{n+s+1} e^{-\delta M} \leq \frac{1}{2}\left|g_{1}-g_{2}\right|_{L^{\infty}(-\infty,-M]}
\end{aligned}
$$

for $M(\rho) \geq \frac{\ln (2 C)+(n+s+1) \ln \rho}{\delta}$. Thus, we obtain $g(x, \zeta)$ satisfying (5.31) and

$$
|g(x, \zeta)| \leq C\left|g_{+}(\zeta)\right|
$$

as a fixed point of the contraction (5.33) on $L^{\infty}(-\infty,-M(\rho)]$. As a uniform limit of iterates $g^{k}$, starting with $g^{0}=0$, that are analytic in $(\lambda, \eta)$ for $\gamma>0$ with continuous extensions to $\gamma \geq 0, g$ inherits the same regularity in $\zeta$. By taking $g_{1}=g$ and $g_{2}=0$ in the first two lines of (5.39) we obtain

$$
\left|\left(g-g_{+}\right)(x, \zeta)\right|=|(\mathcal{T} g-\mathcal{T} 0)(x, \zeta)| \leq C \rho^{n+s+1} e^{\delta x}|g|_{L_{(-\infty,-M)}^{\infty}} \leq C \rho^{n+s+1} e^{\delta x}\left|g_{+}(\zeta)\right| .
$$

This implies (5.27) since $\left|g_{+}(\zeta)\right| \leq C$. The inequality (5.28) is immediate from (5.27).

\section{Stability determinants for the physical equations}

\subsection{Computation of $\Delta_{Z N D}$ and $\Delta_{C J}$.}

First, recall the expressions for $\Delta_{Z N D}$ given in (4.60), (4.62). For $\hat{\gamma}>0$ let

$$
\mathcal{H}(\hat{\zeta}):=-A^{d}\left(w^{*}\right) \mathcal{G}_{1}\left(w^{*}, \hat{\zeta}\right)\left(A^{d}\left(w^{*}\right)\right)^{-1}=\left(\hat{\lambda} A^{0}\left(w^{*}\right)+\sum_{j=1}^{d-1} i \hat{\eta}_{j} A^{j}\left(w^{*}\right)\right)\left(A^{d}\left(w^{*}\right)\right)^{-1}
$$

so that $\left\{R_{-}^{j}(\zeta)\right\}_{j=1}^{n-1+s}$ is now a basis for the negative generalized eigenspace of $\mathcal{H}(\hat{\zeta})$. Since $\theta^{*}(\hat{\zeta})$ is a left eigenvector of $\mathcal{G}_{1}\left(w^{*}, \hat{\zeta}\right)$ associated to the unique eigenvalue with negative real part,

$$
L_{-}(\hat{\zeta}):=\theta^{*}(\hat{\zeta})\left(A^{d}\left(w^{*}\right)\right)^{-1}
$$

is a left eigenvector of $\mathcal{H}(\hat{\zeta})$ associated to the unique eigenvalue $\beta(\hat{\zeta})$ with $\Re \beta(\hat{\zeta})>0$. Using (4.62) we can write 


$$
\Delta_{Z N D}(\hat{\zeta})=\operatorname{det} A^{d}\left(w^{*}\right) L_{-}(\hat{\zeta}) \cdot\left(\hat{\lambda}\left[F^{0}\left(w_{v N}\right)\right]+\sum_{j=1}^{d-1} i \hat{\eta}_{j}\left[F^{j}\left(w_{v N}\right)\right]\right) .
$$

We make one more reformulation in order to relate the computation to one that is done in $[J L]$. Set

$$
K_{-}(\hat{\zeta}):=\left(A^{0}\left(w^{*}\right)\right)^{-1} \mathcal{H}(\hat{\zeta}) A^{0}\left(w^{*}\right) \quad \text { and } \quad \ell_{-}(\hat{\zeta}):=L_{-}(\hat{\zeta}) A^{0}\left(w^{*}\right) .
$$

We then have that

$$
\Delta_{Z N D}(\hat{\zeta})=\operatorname{det} A^{d}\left(w^{*}\right) \ell_{-}(\hat{\zeta})\left(A^{0}\left(w^{*}\right)\right)^{-1}\left(\hat{\lambda}\left[F^{0}\left(w_{v N}\right)\right]+\sum_{j=1}^{d-1} i \hat{\eta}_{j}\left[F^{j}\left(w_{v N}\right)\right]\right)
$$

where $\ell_{-}(\hat{\zeta})$ is a left eigenvector of $K_{-}(\hat{\zeta})$ corresponding to the eigenvalue $\beta(\hat{\zeta})$.

For the explicit computation in the case of the physical equations it is advantageous to let the state variable be $w=(v, u, S, Y)$ where $v=1 / \rho$ is specific volume, $u$ is the velocity, $S$ is specific entropy and $Y$ is the mass fraction of reactant. In these variables the equations simplify and it is straightforward to compute $\left(A^{0}\left(w^{*}\right)\right)^{-1}$ as well as eigenvalues and eigenvectors of $K_{-}(\hat{\zeta})$.

The splitting of the state vector $w=(V, Y)^{\mathrm{T}}$ into gas-dynamical and reactive components induce block structures of $\left(A^{0}\left(w^{*}\right)\right)^{-1}$ and $K_{-}(\hat{\zeta})$ of the form

$$
\left(A^{0}\right)^{-1}=\left(\begin{array}{cc}
C^{-1} & 0 \\
e_{21} & v I_{s \times s}
\end{array}\right), \quad K_{-}(\hat{\zeta})=\left(\begin{array}{cc}
B(\hat{\zeta}) & 0 \\
f_{21} & \frac{\alpha}{u_{d}} I_{s \times s}
\end{array}\right) .
$$

Here $\alpha=\hat{\lambda}+i \hat{\eta} \cdot \tilde{u}$, where $\tilde{u}=\left(u_{1}, \ldots, u_{d-1}\right)$ is the transversal velocity, and $C^{-1}$ and $B(\hat{\zeta})$ are the matrices corresponding to the case of a purely gas-dynamical (i.e. non-reactive) shock.

In particular this implies that the calculation of eigenvalues of $K_{-}(\hat{\zeta})$ is identical to the calculation for the non-reactive Euler equations, which was performed in [JL] under "Method 1". The result is that $K_{-}(\hat{\zeta})$ has a $(d+s)$-fold eigenvalue $\alpha / u_{d}$, together with the eigenvalues

$$
\beta_{ \pm}(\hat{\zeta})=\frac{\alpha u_{d} \pm c \sqrt{\alpha^{2}-\mu|\hat{\eta}|^{2}}}{\mu}
$$

Here $c=\sqrt{-v^{2} p_{v}(v, S)}$ is the sound speed, and $\mu=u_{d}^{2}-c^{2}$. The function $\alpha \mapsto \sqrt{\alpha^{2}-\mu|\hat{\eta}|^{2}}$ in (6.3) is taken to have its branch cut along the imaginary axis between $\pm i|\hat{\eta}| \sqrt{|\mu|}$ and such that it takes positive values along the positive real axis.

Since $\Re \hat{\lambda}>0$ and $\beta_{ \pm}$are evaluated at $0-$, where the flow is sub-sonic, it follows that the unstable eigenvalue of $K_{-}(\hat{\zeta})$ is $\beta_{-}(\hat{\zeta})$. Let the corresponding left eigenvector of $K_{-}(\hat{\zeta})$ be denoted by

$$
\ell_{-}(\hat{\zeta})=\left(\xi_{-}(\hat{\zeta}), \chi_{-}(\hat{\zeta})\right) \in \mathbb{R}^{n} \times \mathbb{R}^{s} .
$$

Then $\xi_{-}(\hat{\zeta})$ is the left eigenvector of $B(\hat{\zeta})$ corresponding to $\beta_{-}(\hat{\zeta})$. The fact that both $\Re \beta_{-}(\hat{\zeta})$ and $\Re \hat{\lambda}$ are positive, while $u_{d}<0$, implies that $\chi_{-}(\hat{\zeta})=0$. Recalling that the right 
and left states of the gas-dynamical variable $V$ in a ZND detonation are the same as for a Lax shock for the non-reactive Euler equations, and using the splitting of $F^{j}(V, Y)$ into gas-dynamical and reactive components, we conclude from (6.2) that $\Delta_{Z N D}(\hat{\zeta})$ is identical with the gas-dynamical Lopatinski determinant computed in [JL].

Due to Galilean invariance we may set $\tilde{u}=0$. Evaluating the inner product in (6.2) by using the Rankine-Hugoniot conditions for the von Neumann jump, we arrive at (see [JL] for details)

$$
\Delta_{Z N D}(\hat{\zeta})=-\frac{C(\hat{\zeta}) u_{d}^{2} v^{2}[\rho]}{1-M^{2}}\left\{\kappa \omega s(\omega)+\left(1-M^{2}\right)\left(r|\hat{\eta}|^{2}-\omega^{2}\right)\right\}
$$

where the flow variables are evaluated at $0-$, and where

$$
M=\frac{\left|u_{d}\right|}{c}, \quad \omega=\frac{\hat{\lambda}}{\left|u_{d}\right|}, \quad r=\frac{\rho}{\rho\left(0_{+}\right)}>1,
$$

and

$$
s(\omega)=\omega+\sqrt{M^{2} \omega^{2}+\left(1-M^{2}\right)|\hat{\eta}|^{2}} .
$$

The coefficient $\kappa$ is given in terms of physical quantities by

$$
\kappa=2+(1-r) M^{2} \Gamma
$$

where $\Gamma=v p_{S}(v, S) / T$ is the Gruneisen coefficient. Finally, the coefficient $C(\hat{\zeta})$ is uniformly bounded, as well as uniformly bounded away from zero, as $\hat{\zeta}$ varies over $S_{+}^{d}$.

In order to analyze the zeros of $\Delta_{Z N D}(\hat{\zeta})$ we observe that its expression coincides (up to a non-vanishing multiplicative factor) with that of the Chapman-Jouguet Lopatinski determinant $\Delta_{C J}(\hat{\zeta})$ in [JLW]. The only difference is in the meaning of the symbols: the flow variables in $\Delta_{Z N D}(\hat{\zeta})$ are evaluated at 0 - instead of at $-\infty$, and the compression ratio $r$ is calculated using $\rho(0 \pm)$ (instead of $\rho(0+)$ and $\rho(-\infty)$ ).

The number and locations of the zeros of $\Delta_{C J}(\hat{\zeta})$ in the set $\bar{S}_{+}^{d}$ were listed in [JLW] by using the winding number analysis from [E2] and [JL]. For the details of this argument we refer to [E2], [JL] and [JLW]. The breakdown of the various cases in terms of physical quantities shows that $\Delta_{Z N D}(\hat{\lambda}, \hat{\eta})$ has:

- $\operatorname{aroot}(\hat{\lambda}, \hat{\eta}) \in \bar{S}_{+}^{d}$ with $\operatorname{Re} \hat{\lambda}>0$ if and only if

$$
\text { (a) } \Gamma \geq \frac{1}{r-1} \frac{1+M}{M^{2}}
$$

- at least one $\operatorname{root}(\hat{\lambda}, \hat{\eta}) \in \bar{S}_{+}^{d}$ with $\operatorname{Re} \hat{\lambda}=0$, but no root with with $\operatorname{Re} \hat{\lambda}>0$, if and only if

$$
\text { (b) }\left(\frac{1}{r-1}\right) \frac{1}{M^{2}}-1 \leq \Gamma<\frac{1}{r-1} \frac{1+M}{M^{2}} \text {; }
$$

- no root in $\bar{S}_{+}^{d}$ if and only if

$$
\text { (c) } \Gamma<\left(\frac{1}{r-1}\right) \frac{1}{M^{2}}-1 .
$$


In particular, consider an ideal polytropic gas whose pressure law is given by $p(\rho, e)=$ $\Gamma \rho e$. The Grüneisen coefficient $\Gamma$ in this case is a fixed constant. A direct calculation shows that

$$
\Gamma<\left(\frac{1}{r-1}\right) \frac{1}{M^{2}}-1
$$

Thus, for an ideal polytropic gas $\Delta_{Z N D}(\hat{\lambda}, \hat{\eta})$ has no root in $\bar{S}_{+}^{d}$.

There is a similar breakdown for $\Delta_{C J}(\hat{\zeta})[\mathrm{JLW}]$ and, again, for an ideal polytropic gas $\Delta_{C J}(\hat{\lambda}, \hat{\eta})$ has no root in $\bar{S}_{+}^{d}$

Remark 6.1. 1. This type of calculation has a long history dating back at least to work by D'yakov [Dy] in the 1950s and Erpenbeck [E2] in the 1960s. Their calculations involved a normal-modes stability analysis for shock-wave solutions of the equations of gas dynamics and led to explicit criteria for stability/instability. However, as noted in [BE], these calculations predicted instability in a parameter regime that was at that time inaccessible to experiment. On the other hand, the instability of steady planar detonations has long been observed in experiments. For example, the multidimensional spinning detonation was observed as early as the 1920s [CW1, CW2]. Later experiments beginning in the 1960s confirmed that detonations often have a quite complicated structure including the well-known pulsating or "galloping" front. The book [FD] contains a description of these experimental studies.

Majda and Rosales ([MR], p. 1315) present evidence that spinning and pulsating detonations in mixtures of hydrogen and oxygen $\left(2 \mathrm{H}_{2}+\mathrm{O}_{2}\right)$ are associated with "radiating boundary waves" associated with the weakly unstable regime (b) above.

2. The instability in the case $\Gamma=(1+M) /\left((r-1) M^{2}\right)$ occurs when the transversal frequency $\hat{\eta}$ vanishes, since $\Delta_{Z N D}(\hat{\tau}, \hat{\gamma}, 0)$ vanishes identically when $\kappa=1-M$. We thus have 1 - D, violent instability.

\section{Existence of curved ZND detonation fronts}

In this section we prove the existence of curved ZND fronts by constructing them as multidimensional perturbations of the planar fronts given by the ZND profile $\tilde{w}(x)$. Given an arbitrarily large finite time $T_{0}$ and assuming nonvanishing of $\Delta_{Z N D}$, we show that provided the initial perturbation $v_{0}\left(y^{\prime}, x\right)$ is small enough, a ZND solution with initial data $\tilde{w}(x)+v_{0}\left(y^{\prime}, x\right)$ and with a curved surface of discontinuity exists on the time interval $\left[0, T_{0}\right]$.

The problem we must solve, already described in (2.26)-(2.29), may be written (recall $\left.t=y_{0}\right)$

$$
\begin{aligned}
& \sum_{j=0}^{d-1} A^{j}(w) w_{y_{j}}+A^{d}(w) w_{x}=R(w) \\
& \sum_{j=0}^{d-1} X_{y_{j}}\left[F^{j}(w)\right]_{*}-\left[F^{d}(w)\right]_{*}=0 \text { on } \mathcal{S} \\
& w\left(0, y^{\prime}, x\right)=\tilde{w}(x)+v_{0}\left(y^{\prime}, x\right), X\left(0, y^{\prime}\right)=0,
\end{aligned}
$$


where the free surface $\mathcal{S}$ is defined by $x=X\left(t, y^{\prime}\right)$. This is a hyperbolic transmission problem for the unknowns $(w, X)$.

Letting $\kappa(x)$ be a test function identically one near $x=0$, we flatten the surface with the change of variables $\tilde{x}=x-\kappa(x) X\left(t, y^{\prime}\right)$ and rewrite the problem (dropping tildes and stars):

$$
\begin{aligned}
& \text { (a) } \sum_{j=0}^{d-1} A^{j}(w) w_{y_{j}}+\mathcal{A}^{d}(w, D(\kappa X)) w_{x}=R(w) \text { in } \pm x \geq 0 \\
& \text { (b) } \sum_{j=0}^{d-1} X_{y_{j}}\left[F^{j}(w)\right]-\left[F^{d}(w)\right]=0 \text { on } x=0 \\
& \text { (c) } w\left(0, y^{\prime}, x\right)=\tilde{w}(x)+v_{0}\left(y^{\prime}, x\right), X\left(0, y^{\prime}\right)=0,
\end{aligned}
$$

where

$$
\mathcal{A}^{d}(w, D(\kappa X)):=A^{d}(w)\left(1-\kappa^{\prime}(x) X\right)-\sum_{j=0}^{d-1} \kappa(x) X_{y_{j}} A^{j}(w) .
$$

The reason for the cutoff $\kappa$ is explained in Remark 7.10. The unknown $w$ may be written

$$
w\left(t, y^{\prime}, x\right)=\left\{\begin{array}{l}
w^{+}\left(t, y^{\prime}, x\right), x \geq 0 \\
w^{-}\left(t, y^{\prime}, x\right), x \leq 0
\end{array},\right.
$$

but we'll often suppress the \pm ; the same applies to $\tilde{w}$ and $v_{0}$ and some other functions that appear below.

Notation 7.1. 1. For $T_{0}>0$ let $\Omega_{T_{0}}=\left[0, T_{0}\right] \times \mathbb{R}^{d}$ and set $\Omega_{T_{0}}^{ \pm}=\Omega_{T_{0}} \cap\{ \pm x \geq 0\}$. For $w$ as in (7.4) we say $w \in \mathcal{H}^{p}\left(\Omega_{T_{0}}\right)$ when $w^{ \pm} \in H^{p}\left(\Omega_{T_{0}}^{ \pm}\right)$and define

$$
|w|_{p}:=\left|w^{+}\right|_{H^{p}\left(\Omega_{T_{0}}^{+}\right)}+\left|w^{-}\right|_{H^{p}\left(\Omega_{T_{0}}^{-}\right.} .
$$

2. Set $\omega_{T_{0}}=\Omega_{T_{0}} \cap\{x=0\}$ and for $X\left(t, y^{\prime}\right) \in H^{p}\left(\omega_{T_{0}}\right)$ define

$$
\langle X\rangle_{p}:=|X|_{H^{p}\left(\omega_{T_{0}}\right)}
$$

When both traces $w^{ \pm}\left(t, y^{\prime}, 0\right) \in H^{p}\left(\omega_{T_{0}}\right)$, we write $w\left(t, y^{\prime}, 0\right) \in \mathcal{H}^{p}\left(\omega_{T_{0}}\right)$ and define

$$
\langle w\rangle_{p}:=\left\langle w^{+}\right\rangle_{p}+\left\langle w^{-}\right\rangle_{p}
$$

The next theorem is our main nonlinear existence result. The proof will occupy the next several subsections.

Theorem 7.2. Assume (HO)-(H4) and

$$
\Delta_{Z N D}(\hat{\zeta}) \neq 0 \text { for } \hat{\zeta} \in \bar{S}_{+}^{d} .
$$


Fix $T_{0}>0$, assume $k>\frac{d}{2}+2$, and suppose $v_{0}^{ \pm} \in H^{k+1}\left(\overline{\mathbb{R}}_{ \pm}^{d}\right)$ both vanish to order $k-1$ at $x=0$ :

$$
\partial_{x}^{j} v_{0}^{ \pm}\left(y^{\prime}, 0\right)=0, j=1, \ldots, k-1 .
$$

For $\epsilon\left(v_{0}\right):=\left|v_{0}^{+}\right|_{H^{k+1}\left(\mathbb{R}_{+}^{d}\right)}+\left|v_{0}^{-}\right|_{H^{k+1}\left(\mathbb{R}_{-}^{d}\right)}$ small enough, there exists a unique solution $\left(w\left(t, y^{\prime}, x\right), X\left(t, y^{\prime}\right)\right)$ to the transmission problem (7.2) on $\Omega_{T_{0}}$ with

$$
w-\tilde{w} \in \mathcal{H}^{k}\left(\Omega_{T_{0}}\right),\left.(w-\tilde{w})\right|_{x=0} \in \mathcal{H}^{k}\left(\omega_{T_{0}}\right), X \in H^{k+1}\left(\omega_{T_{0}}\right) .
$$

Remark 7.3. 1. In order to have a piecewise $H^{k}$ solution as above, it is necessary to impose compatibility conditions on the initial data at the "corner" where $t=0$ and $x=0$. To reduce the technicalities we have chosen the simplest form for the compatibility conditions, namely (7.9). One can easily allow initial data that is nonvanishing at $x=0$ with no essential changes in the arguments below (see [Met1], Defn. 4.2.2).

2. The proof will show that the size of the initial perturbation depends on $T_{0}$ and is also limited by the need to satisfy the block structure and uniform Lopatinski conditions at every stage of the iteration.

\subsubsection{Two initial value problems}

In the first step we ignore the transmission condition in (7.2) and solve away the initial data using the classical theory of the initial value problem for Friedrichs symmetrizable systems ([Ma3], Chapter 2). We refer to Appendix A of [JLW] for the construction of a Friedrichs symmetrizer (there called a Kawashima symmetrizer) for the interior ZND equations.

Let us rewrite $(7.2)$ (a) more concisely as

$$
\mathbb{A}(w, X) D w=R(w) .
$$

Choose an extension of $\tilde{w}^{-}(x)$ into $x>0$, labelled $\tilde{w}(x)$ in this section, such that $\tilde{w}(x) \in$ $C^{\infty} \cap L^{\infty}$ and

$$
\mathbb{A}(\tilde{w}, 0) D \tilde{w}=R(\tilde{w}) \text { on } \mathbb{R}_{x} .
$$

This involves solving the ODE (7.12) after modifying $\mathcal{A}^{d}$ and $R(w)$ in, say, $x>1$. The modification can be chosen to maintain the Friedrichs symmetrizability of (7.11). Next choose an extension of $v_{0}^{-}\left(y^{\prime}, x\right)$ into $x>0$, labelled $v_{0}$ in this section, such that $v_{0} \in$ $H^{k+1}\left(\mathbb{R}^{d}\right)$ and

$$
\left|v_{0}\right|_{H^{k+1}\left(\mathbb{R}^{d}\right)} \leq C\left|v_{0}^{-}\right|_{H^{k+1}\left(\mathbb{R}_{-}^{d}\right)} .
$$

With these choices and modifications, solve the initial value problem on the whole space

$$
\begin{aligned}
& \mathbb{A}\left(\tilde{w}+v_{1}, 0\right) D\left(\tilde{w}+v_{1}\right)=R\left(\tilde{w}+v_{1}\right) \\
& \left.v_{1}\right|_{t=0}=v_{0} .
\end{aligned}
$$

A slight modification of the argument of [Ma3], Theorem 2.1, yields, for small enough $\left|v_{0}^{-}\right|_{H^{k+1}\left(\mathbb{R}_{-}^{d}\right)}$, a unique solution

$$
v_{1} \in C\left(\left[-1, T_{0}\right], H^{k+1}\left(\mathbb{R}^{d}\right)\right) \cap C^{1}\left(\left[-1, T_{0}\right], H^{k}\left(\mathbb{R}^{d}\right)\right) \cap H^{k+1}\left(\left[-1, T_{0}\right] \times \mathbb{R}^{d}\right)
$$


with

$$
\left|v_{1}\right|_{N} \leq C\left|v_{0}^{-}\right|_{H^{k+1}\left(\mathbb{R}_{-}^{d}\right)} \leq C \epsilon\left(v_{0}\right)
$$

where $N$ is the natural norm on the space in (7.15).

Similarly, by taking extensions of the original $\tilde{w}^{+}$and $v_{0}^{+}$into $x<0$, define an initial value problem analogous to $(7.14)$ for an unknown $v_{2}$. For small enough $\left|v_{0}^{+}\right|_{H^{k+1}\left(\mathbb{R}_{+}^{d}\right)}$ we obtain a solution $v_{2}$ such that

$$
\left|v_{2}\right|_{N} \leq C\left|v_{0}^{+}\right|_{H^{k+1}\left(\mathbb{R}_{+}^{d}\right)} \leq C \epsilon\left(v_{0}\right)
$$

Finally, define $v \in \mathcal{H}^{k+1}\left(\left[-1, T_{0}\right] \times \mathbb{R}^{d}\right)$ by

$$
v=\left\{\begin{array}{l}
\left.v_{2}\right|_{x \geq 0}, x \geq 0 \\
\left.v_{1}\right|_{x \leq 0}, x \leq 0
\end{array} .\right.
$$

\subsubsection{Forward transmission problem}

We can now reduce to a transmission problem on $\left[-1, T_{0}\right] \times \mathbb{R}^{d}$ where all data is zero in $t<0$. Denote the boundary operator on the left side of $(7.2)(\mathrm{b})$ by $\mathbb{B}(w, X)$ and for $v\left(t, y^{\prime}, x\right)$ as in (7.18) define

$$
\begin{aligned}
& \mu\left(t, y^{\prime}, x\right):=\tilde{w}(x)+v\left(t, y^{\prime}, x\right) \\
& b(\mu):=\left\{\begin{array}{l}
-\mathbb{B}(\mu, 0), t \geq 0 \\
0, t<0
\end{array} \quad \text { on } x=0 .\right.
\end{aligned}
$$

Since

$$
-\mathbb{B}(\mu, 0)=[\phi(\tilde{w}, v) v]
$$

for some smooth $\phi$, the compatibility conditions (7.9) (together with the Moser estimates stated below) imply

$$
b(\mu) \in H^{k}\left(\left[-1, T_{0}\right] \times \mathbb{R}^{d-1}\right) \text { with }|b(\mu)|_{k} \leq C \epsilon\left(v_{0}\right) .
$$

We shall look for a solution $(w, X)$ to $(7.2)$ where

$$
w=\mu+z
$$

for a new unknown $z$. If we define $\mathcal{B}(\mu, z)$ by

$$
\begin{aligned}
& \mathcal{B}(\mu, z)(z, \partial X):= \\
& \mathbb{B}(\mu+z, X)-\mathbb{B}(\mu, 0)=\sum_{j=0}^{d-1} X_{y_{j}}\left[F^{j}(\mu+z)\right]-\left[\left(\int_{0}^{1} A^{d}(\mu+s z) d s\right) z\right],
\end{aligned}
$$


we see that $(w, X)$ is a solution to $(7.2)$ on $\Omega_{T_{0}}$ if and only if $(z, X)$ satisfies the following forward transmission problem on $\left[-1, T_{0}\right] \times \mathbb{R}^{d}$ :

$$
\begin{aligned}
& \mathbb{A}(\mu+z, X) D z=(\mathbb{A}(\mu, 0)-\mathbb{A}(\mu+z, X)) D \mu+R(\mu+z)-R(\mu) \\
& \mathcal{B}(\mu, z)(z, \partial X)=b(\mu) \text { on } x=0 \\
& z=0, X=0 \text { in } t<0 .
\end{aligned}
$$

We'll solve (7.25) by the iteration scheme

$$
\begin{aligned}
& \text { (a) } \mathbb{A}\left(\mu+z_{n}, X_{n}\right) D z_{n+1}=\left(\mathbb{A}(\mu, 0)-\mathbb{A}\left(\mu+z_{n}, X_{n}\right)\right) D \mu+R\left(\mu+z_{n}\right)-R(\mu) \\
& \text { (b) } \mathcal{B}\left(\mu, z_{n}\right)\left(z_{n+1}, \partial X_{n+1}\right)=b(\mu) \text { on } x=0 \\
& \text { (c) } z_{n+1}=0, X_{n+1}=0 \text { in } t<0
\end{aligned}
$$

where $z_{0}=0, X_{0}=0$.

\subsubsection{Norms for iteration; induction step.}

First we define the norms used to prove convergence of the scheme (7.25). Set $\mathcal{O}:=$ $\left[-1, T_{0}\right] \times \mathbb{R}^{d}$ and $b \mathcal{O}:=\mathcal{O} \cap\{x=0\}$.

Definition 7.4. 1. Let $\partial=\left(\partial_{t}, \partial_{y^{\prime}}\right)$. For $p$ a nonnegative integer and $\gamma \geq 1$ set

$$
\begin{aligned}
|z|_{p, \gamma} & :=\sum_{j=0}^{p} \gamma^{p-j}\left|e^{-\gamma t} \partial^{j} z\right|_{L^{2}(\mathcal{O})} \\
\langle z\rangle_{p, \gamma} & =\sum_{j=0}^{p} \gamma^{p-j}\left|e^{-\gamma t} \partial^{j} z\right|_{L^{2}(b \mathcal{O})},
\end{aligned}
$$

where the sum over $z^{ \pm}$in both cases is understood. $\langle X\rangle_{p, \gamma}$ is defined similarly.

2. Let $D=\left(\partial_{t}, \partial_{y^{\prime}}, \partial_{x}\right)$. Set $|u|_{*}:=|u|_{L^{\infty}(\mathcal{O})}$ and let

$$
\|z, X\|^{*}:=\left|z, D z, X, \partial X, \partial^{2} X\right|_{*} .
$$

3. Define

$$
\begin{aligned}
\|z, X\|_{p, \gamma}^{\prime} & :=|z|_{p, \gamma}+\langle z, X, \partial X\rangle_{p, \gamma} \\
\|z, X\|_{p, \gamma} & :=\|z, X\|_{p, \gamma}^{\prime}+\left|\partial_{x} z\right|_{p-1, \gamma}+\left|\partial_{x}^{2} z\right|_{p-2, \gamma} .
\end{aligned}
$$

Observe that

$$
|z|_{p-1, \gamma} \leq \frac{1}{\gamma}|z|_{p, \gamma}
$$

The following version of the standard Sobolev estimate will also be useful:

$$
\text { Sobolev estimate: } \quad|z|_{*} \leq C(\gamma)\left(|z|_{p, \gamma}+\left|\partial_{x} z\right|_{p, \gamma}\right) \text { for } p>\frac{d}{2}
$$

From (7.30) we deduce the estimate

$$
\|z, X\|^{*} \leq C_{1}(\gamma)\|z, X\|_{k, \gamma} \text { for } k>\frac{d}{2}+2 .
$$

The following Proposition, proved below, is the basis for the induction argument. 
Proposition 7.5 (Higher derivative estimate). Consider the linear transmission problem (7.25) for the unknown $\left(z_{n+1}, X_{n+1}\right)$. Assume $k \geq 0$, let $\epsilon\left(v_{0}\right)$ be as in Theorem 7.2, and recall $\mu=\tilde{w}+v$. There exist positive constants $\delta$, $\gamma_{0}$ such that if

$$
\epsilon\left(v_{0}\right)<\delta \text { and }\left\|z_{n}, X_{n}\right\|^{*}<\delta,
$$

then for $\gamma \geq \gamma_{0}$ the unique solution of (7.25) satisfies

$$
\begin{aligned}
& \left\|z_{n+1}, X_{n+1}\right\|_{k, \gamma} \leq \\
& \frac{C(k)}{\sqrt{\gamma}}\left\|z_{n}, X_{n}\right\|_{k, \gamma}+C(k)\left\|z_{n+1}, X_{n+1}\right\|^{*}\left(\left\|z_{n}, X_{n}\right\|_{k, \gamma}+\|v\|_{k, \gamma}\right)+C_{2}(\gamma) \Phi_{k}\left(\epsilon\left(v_{0}\right)\right),
\end{aligned}
$$

where $\Phi_{k}(s)$ is a continuous increasing function with $\Phi_{k}(0)=0$.

Assuming the above Proposition for a moment, we prove

Proposition 7.6 (High norm boundedness). Let $k>\frac{d}{2}+2$ and let $\gamma_{0}, \delta$, and $C_{2}(\gamma)$ be as in Prop. 7.5. There exist constants $\gamma_{1} \geq \gamma_{0}$ and $\delta_{1}(\gamma) \leq \delta$ such that for $\gamma \geq \gamma_{1}$ and $\epsilon\left(v_{0}\right)<\delta_{1}(\gamma)$ we have for all $n$

$$
\begin{aligned}
& \text { (a) }\left\|z_{n}, X_{n}\right\|^{*}<\delta \\
& \text { (b) }\left\|z_{n}, X_{n}\right\|_{k, \gamma} \leq 2 C_{2}(\gamma) \Phi_{k}\left(\epsilon\left(v_{0}\right)\right) .
\end{aligned}
$$

Proof. The statement holds for $n=0$; assume it holds for a given $n$. Let $a_{n}=\left\|z_{n}, X_{n}\right\|_{k, \gamma}$. Together with $\|v\|_{k, \gamma} \leq C(k) \epsilon\left(v_{0}\right)$, the estimates (7.31), (7.33), and (7.34)(b) imply

$$
\begin{aligned}
& a_{n+1} \leq \frac{C(k)}{\sqrt{\gamma}} 2 C_{2}(\gamma) \Phi_{k}\left(\epsilon\left(v_{0}\right)\right)+ \\
& \quad C(k) C_{1}(\gamma) a_{n+1}\left[2 C_{2}(\gamma) \Phi_{k}\left(\epsilon\left(v_{0}\right)\right)+\epsilon\left(v_{0}\right)\right]+C_{2}(\gamma) \Phi_{k}\left(\epsilon\left(v_{0}\right)\right) .
\end{aligned}
$$

First choose $\gamma_{1}$ such that $2 C(k) / \sqrt{\gamma_{1}} \leq \frac{1}{3}$. Then for any $\gamma \geq \gamma_{1}$ choose $\delta_{1}(\gamma) \leq \delta$ such that

$$
C(k) C_{1}(\gamma)\left[2 C_{2}(\gamma) \Phi_{k}\left(\epsilon\left(v_{0}\right)\right)+\epsilon\left(v_{0}\right)\right] \leq \frac{1}{3} \text { for } \epsilon\left(v_{0}\right)<\delta_{1}(\gamma)
$$

Thus for $\gamma \geq \gamma_{1}$ and $\epsilon\left(v_{0}\right)<\delta_{1}(\gamma)$ we have

$$
a_{n+1} \leq \frac{1}{3} C_{2}(\gamma) \Phi_{k}\left(\epsilon\left(v_{0}\right)\right)+\frac{1}{3} a_{n+1}+C_{2}(\gamma) \Phi_{k}\left(\epsilon\left(v_{0}\right)\right),
$$

which is equivalent to $(7.34)$ (b) for $n+1$ :

$$
\left\|z_{n+1}, X_{n+1}\right\|_{k, \gamma} \leq 2 C_{2}(\gamma) \Phi_{k}\left(\epsilon\left(v_{0}\right)\right) .
$$

Applying (7.31) and (7.38) and reducing $\delta_{1}(\gamma)$ if necessary, we arrange (7.34)(a) for $n+1$. The choices of $\gamma_{1}$ and $\delta_{1}(\gamma)$ are independent of $n$. 


\subsection{4 $\Delta_{Z N D}$ and the main linear estimate.}

In this section we discuss the $L^{2}$ estimate for the linearized transmission problem on which the proof of Proposition 7.5 is based. In the process we'll clarify the role of $\Delta_{Z N D}(4.60)$, (4.62) in the argument.

With $\mu=\tilde{w}+v, \mathbb{A}$, and $\mathcal{B}$ as in (7.25), we consider the following linear problem for the unknown $(z, X)$ :

$$
\begin{aligned}
& \text { (a) } \mathbb{A}(\mu+\tilde{z}, \tilde{X}) D z=f \text { on } \mathcal{O} \\
& \text { (b) } \mathcal{B}(\mu, \tilde{z})(z, \partial X)=g \text { on } b \mathcal{O} \\
& \text { (c) } z=0, X=0 \text { in } t<0 .
\end{aligned}
$$

Proposition 7.7 ( $L^{2}$ estimate). Assume $f \in L^{2}(\mathcal{O})$ and $g \in L^{2}(b \mathcal{O})$ both vanish in $t<0$, and assume

$$
\Delta_{Z N D}(\hat{\zeta}) \neq 0 \text { on } \bar{S}_{+}^{d}
$$

Fix $K>0$ and suppose $\|v, \tilde{z}, \tilde{X}\|^{*} \leq K$. There exists a positive constant $\delta_{0}$ such that if

$$
|v, \tilde{z}, \tilde{X}, \partial \tilde{X}|_{*} \leq \delta_{0}
$$

then the problem (7.39) has a unique solution $(z, X) \in L^{2}(\mathcal{O}) \times H^{1}(b \mathcal{O})$. Moreover, there exist positive constants $C$ and $\gamma_{0}$ such that for $\gamma \geq \gamma_{0}$ :

$$
\gamma|z|_{0, \gamma}^{2}+\langle z, \gamma X, \partial X\rangle_{0, \gamma}^{2} \leq C\left(\frac{1}{\gamma}|f|_{0, \gamma}^{2}+\langle g\rangle_{0, \gamma}^{2}\right) .
$$

Proof. 1. We shall carry the proof to the point where results of [Met1, Met2] can be applied. It will be helpful to have the explicit formulas for $\mathbb{A}$ and $\mathcal{B}$ :

$$
\begin{aligned}
& \mathbb{A}(\mu+\tilde{z}, \tilde{X}) D z=\sum_{j=0}^{d-1} A^{j}(\mu+\tilde{z}) \partial_{j} z+\left(A^{d}(\mu+\tilde{z})\left(1-\kappa^{\prime}(x) \tilde{X}\right)-\sum_{j=0}^{d-1} \kappa(x) \tilde{X}_{y_{j}} A^{j}(\mu+\tilde{z})\right) \partial_{x} z \\
& \mathcal{B}(\mu, \tilde{z})(z, \partial X)=\sum_{j=0}^{d-1} X_{y_{j}}\left[F^{j}(\mu+\tilde{z})\right]-\left[\left(\int_{0}^{1} A^{d}(\mu+s \tilde{z}) d s\right) z\right] .
\end{aligned}
$$

2. The main step in the proof of Proposition 7.7 is to obtain an a priori estimate of the form (7.42) for $C^{\infty}$ compactly supported $z$ and $X$, where $f$ and $g$ are now defined by the left sides of (7.39)(a),(b). The result then follows by a duality argument as in [Met1], Theorem 3.1.1.

For $z$ supported away from $x=0$, the estimate

$$
\gamma|z|_{0, \gamma}^{2} \leq \frac{C}{\gamma}|f|_{0, \gamma}^{2}
$$

can be proved using the Friedrichs symmetrizability of $\mathbb{A}$ by a simple integration by parts as in $[\mathrm{CP}]$, Chpt. 7, Thm. 4.1. In this case the estimate

$$
\langle\gamma X, \partial X\rangle_{0, \gamma} \leq C\langle g\rangle_{0, \gamma}
$$


follows directly, when $\delta_{0}$ in (7.41) is small enough, from the linear independence of the jumps $\left[F^{j}\left(w_{v N}\right)\right], j=0, \ldots, d-1$. That in turn is a consequence of $(7.40)$; the last column of (4.60) is nonvanishing for all $\hat{\zeta} \in \bar{S}_{+}^{d}$.

The argument is much more involved in the case when $z$ has support near $x=0$, and requires a Kreiss symmetrizer $[\mathrm{K}]$ of the type first constructed for shock problems in [Ma1]. Two conditions are needed for the construction of the symbol of the paradifferential symmetrizer: the block structure condition ([Met2], Defn. 1.2), which is a condition on the frozen coefficient matrices $\mathcal{G}_{1}\left(q_{ \pm}, \hat{\zeta}\right)$ defined below, and the uniform Lopatinski condition.

3. Uniform Lopatinski condition. Consider again the definition of $\Delta_{Z N D}$. With $\mathcal{B}$ as in (7.39) the boundary condition in (4.56) may be written

$$
\mathcal{B}\left(w_{v N}, 0\right)(w, d \phi)=\sum_{j=0}^{d-1} \partial_{j} \phi\left[F^{j}\left(w_{v N}\right)\right]-\left[A^{d}\left(w_{v N}\right) w\right]=0 .
$$

Define $\hat{\mathcal{B}}$ using the boundary condition in the transformed problem (4.57):

$$
\hat{\mathcal{B}}\left(w_{v N}, 0 ; \hat{\zeta}\right)(\hat{w}, \hat{\phi}):=\hat{\phi}\left(\hat{\lambda}\left[F^{0}\left(w_{v N}\right)\right]+\sum_{j=1}^{d-1} i \hat{\eta}_{j}\left[F^{j}\left(w_{v N}\right)\right]\right)-\left[A^{d}\left(w_{v N}\right) \hat{w}\right] .
$$

Letting $\mathcal{E}^{-}\left(w^{*}, \hat{\zeta}\right)$ denote the $n-1+s$ dimensional decaying generalized eigenspace of $\mathcal{G}_{1}\left(w^{*}, \hat{\zeta}\right)$, we may rephrase assumption (7.40) in the equivalent form

$$
\hat{\mathcal{B}}\left(w_{v N}, 0 ; \hat{\zeta}\right): \mathcal{E}^{-}\left(w^{*}, \hat{\zeta}\right) \times \mathbb{C} \rightarrow \mathbb{C}^{n+s} \text { is an isomorphism for all } \hat{\zeta} \in \bar{S}_{+}^{d} .
$$

Denote by $\mathbb{A}\left(q_{ \pm}\right) D$ (resp., $\mathcal{B}\left(q_{+}^{0}, q_{-}^{0}\right)$ the constant coefficient operators obtained by freezing

$$
\left(\mu+\tilde{z}-w_{v N}, \kappa^{\prime} \tilde{X}, \kappa \partial \tilde{X}\right)\left(\operatorname{resp} .,\left(\mu-w_{v N}, \tilde{z}\right)\right)
$$

in (7.43), where the subscripts on $q$ (resp., $q^{0}$ ) distinguish the cases $\pm x \geq 0$ (resp., right and left sided limits at $x=0)$. Parallel to $(4.57)$ we now Fourier-Laplace transform the frozen problem. Define $\mathcal{G}_{1}\left(q_{ \pm}, \hat{\zeta}\right)$ as in (4.57) by rewriting the transformed problem with $z^{\prime}$ on the left and let $\mathcal{E}^{-}\left(q_{ \pm}, \hat{\zeta}\right)$ be the corresponding decaying (for $\pm x \geq 0$ ) generalized eigenspaces. By continuity (7.48) implies that there exists a $\delta_{0}>0$ such that for

$$
\left|q_{+}, q_{-}, q_{+}^{0}, q_{-}^{0}\right| \leq \delta_{0}
$$

$\mathcal{E}^{-}\left(q_{+}, \hat{\zeta}\right)$ is trivial, $\mathcal{E}^{-}\left(q_{-}, \hat{\zeta}\right)$ is $n-1+s$ dimensional, and

$$
\hat{\mathcal{B}}\left(q_{+}^{0}, q_{-}^{0} ; \hat{\zeta}\right): \mathcal{E}^{-}\left(q_{-}, \hat{\zeta}\right) \times \mathbb{C} \rightarrow \mathbb{C}^{n+s} \text { is an isomorphism for all } \hat{\zeta} \in \bar{S}_{+}^{d},
$$

where $\hat{\mathcal{B}}\left(q_{+}^{0}, q_{-}^{0} ; \hat{\zeta}\right)$ is the obvious analogue of the operator in (7.48). Condition (7.51) is called the uniform Lopatinski condition for the problem (7.39).

4. Block structure. Theorem 1.3 of [Met2] implies that when $\mathbb{A}(\mu+\tilde{z}, \partial \tilde{X}) D$ defines a symmetrizable hyperbolic system with noncharacteristic boundary matrix $\mathcal{A}^{d}(7.3)$ and characteristics of constant multiplicity, the block structure condition is satisfied. Thus, our 
assumptions (H0), (H1), and (H3) imply that for $\delta_{0}$ (7.50) small enough, the block structure condition is satisfied by the matrices $\mathcal{G}_{1}\left(q_{ \pm}, \hat{\zeta}\right)$.

The a priori estimate (7.42) is now proved for $z$ supported sufficiently near $x=0$ as in [Met1], Thm. 2.1.3. The Lipschitz condition $\|v, \tilde{z}, \tilde{X}\|^{*} \leq K$ provides the regularity needed to use the paradifferential calculus to transfer the argument from the symbolic level to the operator level.

Remark 7.8. In the proof of Proposition 7.5 we shall use the following slightly weaker $L^{2}$ estimate for solutions of (7.39):

$$
|z|_{0, \gamma}+\langle z, X, \partial X\rangle_{0, \gamma} \leq C\left(\frac{1}{\sqrt{\gamma}}|f|_{0, \gamma}+\langle g\rangle_{0, \gamma}\right)
$$

\subsubsection{Higher derivative estimates.}

Proof of Proposition 7.5.

1. Preliminaries. We recall that $\mu=\tilde{w}+v$ for $v$ as in (7.18). In view of (7.16), (7.17), any norm of $v$ that appears below is dominated by $C(k) \epsilon\left(v_{0}\right)$. Observe that the case $k=0$ follows directly from (7.21) and (7.52).

In the argument below $\Phi=\Phi\left(v, D v, z_{n}\right)$ will denote a $C^{\infty}$ function, which may change from term to term, of $\tilde{w}, \tilde{w}^{\prime}$ and the arguments shown. An expression like

$$
\Phi=\Phi\left(v, D v, z_{n}\right)(z, \partial X)
$$

indicates linear dependence on the last two arguments.

The main extra tool we need for the higher derivative estimates is the following weighted version of the standard Moser estimate ([G], Lemma 2.1.2).

Lemma 7.9 (Moser estimates). For $k \in \mathbb{N}=\{0,1,2, \ldots\}$ let $\alpha_{1}+\cdots+\alpha_{r} \leq j \leq k$, $\alpha_{i} \in \mathbb{N}$. Then

$$
\gamma^{k-j}\left|\left(\partial^{\alpha_{1}} w_{1}\right) \cdots\left(\partial^{\alpha_{r}} w_{r}\right)\right|_{0, \gamma} \leq C \sum_{i=1}^{r}\left|w_{i}\right|_{k, \gamma}\left(\prod_{j \neq i}\left|w_{j}\right|_{*}\right) .
$$

Remark 7.10. If $\kappa_{1}(x)$ is any $C^{\infty}$ compactly supported function such that $\kappa_{1} \kappa=\kappa$, we have

$$
\mathcal{A}^{d}(w, D(\kappa X))=\mathcal{A}^{d}\left(w, D\left(\kappa \kappa_{1} X\right)\right)
$$

Thus, in the following estimates we may replace all front terms, $X_{n}$ or $X_{n+1}$, by $\kappa_{1} X_{n}$ or $\kappa_{1} X_{n+1}$. Although we suppress the $\kappa_{1}$ below, we shall always make this replacement. With this convention observe that the $L^{2}$ estimate (7.52) gives exactly the same interior and trace control over $(X, \partial X)$ as it does over $z$. This allows us to treat $z$ terms and front terms in the same way in both interior and boundary Moser estimates. 
2. Tangential higher derivative estimates. The first step in proving (7.33) is to show

$$
\begin{aligned}
& \left\|z_{n+1}, X_{n+1}\right\|_{k, \gamma}^{\prime} \leq \frac{C(k)}{\sqrt{\gamma}}\left\|z_{n+1}, X_{n+1}\right\|_{k, \gamma}+ \\
& \frac{C(k)}{\sqrt{\gamma}}\left\|z_{n}, X_{n}\right\|_{k, \gamma}+C(k)\left\|z_{n+1}, X_{n+1}\right\|^{*}\left(\left\|z_{n}, X_{n}\right\|_{k, \gamma}+\|v\|_{k, \gamma}\right)+C_{2}(\gamma) \Phi_{k}\left(\epsilon\left(v_{0}\right)\right),
\end{aligned}
$$

where $\left\|z_{n+1}, X_{n+1}\right\|_{k, \gamma}^{\prime}$ as in (7.28).

Let us write the interior equation $(7.25)$ (a) as

$$
\mathbb{A}\left(\mu+z_{n}, X_{n}\right) z_{n+1}=\Phi\left(v, D v, z_{n}\right)\left(z_{n}, \partial X_{n}\right):=f_{n} .
$$

To estimate $\left\|z_{n+1}, X_{n+1}\right\|_{k, \mu, \gamma}^{\prime}$ we apply the $L^{2}$ estimate (7.52) to the problem satisfied by $\gamma^{k-j} \partial^{j}\left(z_{n+1}, X_{n+1}\right), j \leq k$ and use (7.54) to estimate products. We omit the details since the procedure is by now rather standard [Met1, GMWZ].

4. Normal derivative estimates. We still need to estimate $\left|\partial_{x} z_{n+1}\right|_{k-1, \gamma}$ and $\left|\partial_{x}^{2} z_{n+1}\right|_{k-2, \gamma}$. Let

$$
\Psi=\Psi\left(v, D v, z_{n}, X_{n}, \partial X_{n}\right)
$$

denote a $C^{\infty}$ function, which may change from term to term, of $\tilde{w}, \tilde{w}^{\prime}$ and the arguments shown. Noting that $\mathcal{A}^{d}\left(x, \mu+z_{n}, X_{n}, \partial X_{n}\right)$ (7.3) is invertible when $\delta$ in (7.32) is small enough, we may rewrite the interior equation (7.25)(a) as

$$
\partial_{x} z_{n+1}=\Psi\left(v, D v, z_{n}, X_{n}, \partial X_{n}\right)\left(z_{n}, \partial X_{n}\right)+\Psi\left(v, D v, z_{n}, X_{n}, \partial X_{n}\right) \partial z_{n+1} .
$$

To estimate $\left|\partial_{x} z_{n+1}\right|_{k-1, \gamma}$ we use the Moser estimates together with the estimate of $\left|z_{n+1}\right|_{k, \gamma}$ from above. The estimate of $\left|\partial_{x}^{2} z_{n+1}\right|_{k-2, \gamma}$ is obtained by differentiating (7.59) and is similar to the previous step.

\subsubsection{Contraction in the $L^{2}$ norm.}

In this section we finish the proof of Theorem 7.2. Setting

$$
Z_{n}:=\left(z_{n}, X_{n}, \partial X_{n}\right)
$$

and considering the difference of the problems (7.25) satisfied by $Z_{n}$ and $Z_{n+1}$ we find

$$
\begin{aligned}
& \mathbb{A}\left(\mu+z_{n}, X_{n}\right) D\left(z_{n+1}-z_{n}\right)=\psi_{1}\left(v, D v, D z_{n}, Z_{n}, Z_{n-1}\right)\left(Z_{n}-Z_{n-1}\right) \\
& \mathcal{B}\left(\mu, z_{n}\right)\left(z_{n+1}-z_{n}, \partial X_{n+1}-\partial X_{n}\right)=\psi_{2}\left(v, z_{n-1}, Z_{n}\right)\left(z_{n}-z_{n-1}\right):= \\
& -\left(\mathcal{B}\left(\mu, z_{n}\right)-\mathcal{B}\left(\mu, z_{n-1}\right)\right)\left(z_{n}, \partial X_{n}\right), \\
& z_{n+1}-z_{n}=0, X_{n+1}-X_{n}=0 \text { in } t<0
\end{aligned}
$$

where $\psi_{1}$ and $\psi_{2}$ are smooth functions of $\tilde{w}, \tilde{w}^{\prime}$ and the arguments shown.

The iterates satisfy the uniform estimates (7.34) for a $k$ such that $k>\frac{d}{2}+2$. Set

$$
\zeta_{n+1}:=Z_{n+1}-Z_{n} \text { and }||\left|\zeta_{n}\right| \|_{0, \gamma}:=\left|\zeta_{n}\right|_{0, \gamma}+\left\langle\zeta_{n}\right\rangle_{0, \gamma}
$$


and apply the $L^{2}$ estimate (7.52) to (7.61) to obtain

$$
\left|\left\|\zeta _ { n + 1 } \left|\left\|_{0, \gamma} \leq C\left(\frac{1}{\sqrt{\gamma}}\left|Z_{n}-Z_{n-1}\right|_{0, \gamma}+\left|Z_{n}\right|_{*}\left\langle z_{n}-z_{n_{1}}\right\rangle_{0, \gamma}\right) \leq C\left(\frac{1}{\sqrt{\gamma}}+\left|Z_{n}\right|_{*}\right)||\left|\zeta_{n}\right|\right\|_{0, \gamma},\right.\right.\right.
$$

where we have used the special form of $\psi_{2}$. Since we can estimate $\left|Z_{n}\right|_{*}$ by the Sobolev estimate (7.31), we conclude from (7.63) and (7.34)(b) that the iterates $Z_{n}$ converge in the $\left|\|\cdot \mid\|_{0, \gamma}\right.$ norm, for a fixed $\gamma \geq \gamma_{1}$ large enough and $0<\epsilon\left(v_{0}\right)<\delta_{1}(\gamma)$ small enough, to some $(z, X, \partial X)$. In view of the estimates (7.34), a standard argument using interpolation and weak convergence shows that $(z, X)$ satisfies the nonlinear error problem (7.24) and satisfies the estimates (7.34).

This concludes the proof of Theorem 7.2.

Remark 7.11. 1. The key role of $\Delta_{Z N D}$ in this finite time existence question might be understood vaguely as follows. Finite time existence of slightly perturbed fronts is governed by stability in the high frequency regime. High frequency perturbations encountering the von Neumann jump cannot "distinguish" $\tilde{w}(x)$ from $w^{*}$. Taken together, Theorems 5.1 and 7.2 support these statements.

2. As a check on the validity of the physical ZND equations, it is worthwhile to show that the $Y$-component of the solution $w$ derived in Theorem 7.2 remains physically meaningful, $0 \leq Y \leq 1$, for all times during the interval of existence $\left[0, T_{0}\right]$, provided this is true at time zero. This is shown in [Cos] by analyzing the reaction equation and using the observation that $Y$ remains continuous across the front $\mathcal{S}$. The latter property may be deduced from the Rankine-Hugoniot conditions together with the fact that $g^{j}(V)$ is equal to the first component of $f^{j}(V)$ in the case of the physical equations.

3. Short time existence results for curved ZND and CJ fronts are proved by similar methods in [Cos]. There the size of the initial perturbation is limited only by the need to satisfy the block structure and uniform Lopatinski conditions at every stage of the iteration.

\section{Appendix: Curved fronts in the Chapman-Jouguet model}

Consider now the Chapman-Jouguet system (2.30)-(2.34) for the unknowns $(V, X)$. With $\tilde{v}$ as in (2.18) and $\tilde{a}^{j}=d \tilde{f}^{j}$ we consider, parallel to (7.2) the equivalent fixed-boundary transmission problem

(a) $\sum_{j=0}^{d-1} \tilde{a}^{j}(V) V_{y_{j}}+\tilde{\mathcal{A}}^{d}(V, D(\kappa X)) V_{x}=0$ in $\pm x \geq 0$

(b) $\sum_{j=0}^{d-1} X_{y_{j}}\left[\tilde{f}^{j}(V)\right]-\left[\tilde{f}^{d}(V)\right]=0$ on $x=0$

(c) $V\left(0, y^{\prime}, x\right)=\tilde{v}+v_{0}\left(y^{\prime}, x\right), X\left(0, y^{\prime}\right)=0$,

where

$$
\tilde{\mathcal{A}}^{d}(V, D(\kappa X)):=\tilde{a}^{d}(V)\left(1-\kappa^{\prime}(x) X\right)-\sum_{j=0}^{d-1} \kappa(x) X_{y_{j}} \tilde{a}^{j}(V) .
$$


Theorem 8.1. Assume (HO)-(H4) and

$$
\Delta_{C J}(\hat{\zeta}) \neq 0 \text { for } \hat{\zeta} \in \bar{S}_{+}^{d} .
$$

Fix $T_{0}>0$, assume $k>\frac{d}{2}+2$, and suppose $v_{0}^{ \pm} \in H^{k+1}\left(\overline{\mathbb{R}}_{ \pm}^{d}\right)$ both vanish to order $k-1$ at $x=0$ :

$$
\partial_{x}^{j} v_{0}^{ \pm}\left(y^{\prime}, 0\right)=0, j=1, \ldots, k-1 .
$$

For $\epsilon\left(v_{0}\right):=\left|v_{0}^{+}\right|_{H^{k+1}\left(\mathbb{R}_{+}^{d}\right)}+\left|v_{0}^{-}\right|_{H^{k+1}\left(\mathbb{R}_{-}^{d}\right)}$ small enough, there exists a unique solution $\left(V\left(t, y^{\prime}, x\right), X\left(t, y^{\prime}\right)\right)$ to the transmission problem (8.1) on $\Omega_{T_{0}}$ with

$$
V-\tilde{v} \in \mathcal{H}^{k}\left(\Omega_{T_{0}}\right),\left.(V-\tilde{v})\right|_{x=0} \in \mathcal{H}^{k}\left(\omega_{T_{0}}\right), X \in H^{k+1}\left(\omega_{T_{0}}\right) .
$$

Proof. 1. Preliminaries. The proof is almost identical to that of Theorem 7.2 , but there are a few things to check because now, in contrast to the ZND system, the definition of the fluxes $\tilde{f}^{j}$ changes discontinuously across the front (recall Remark 2.7). We construct the $n$-vector $V$ of the form

$$
V\left(t, y^{\prime}, x\right)=\tilde{v}+v\left(t, y^{\prime}, x\right)+z\left(t, y^{\prime}, x\right)=\mu\left(t, y^{\prime}, x\right)+z\left(t, y^{\prime}, x\right)
$$

for $\tilde{v}$ as in (2.18), where $v$ is defined as in (7.18) using the solutions $\left(v_{1}, v_{2}\right)$ to a pair of initial value problems, and $z$ is the solution to a forward transmission problem. In place of (7.43) we have now

$$
\begin{aligned}
& \mathbb{A}(\mu+\tilde{z}, \tilde{X}) D z=\sum_{j=0}^{d-1} \tilde{a}^{j}(\mu+\tilde{z}) \partial_{j} z+\left(\tilde{a}^{d}(\mu+\tilde{z})\left(1-\kappa^{\prime}(x) \tilde{X}\right)-\sum_{j=0}^{d-1} \kappa(x) \tilde{X}_{y_{j}} \tilde{a}^{j}(\mu+\tilde{z})\right) \partial_{x} z \\
& \mathcal{B}(\mu, \tilde{z})(z, \partial X)=\sum_{j=0}^{d-1} X_{y_{j}}\left[\tilde{f}^{j}(\mu+\tilde{z})\right]-\left[\left(\int_{0}^{1} \tilde{a}^{d}(\mu+s \tilde{z}) d s\right) z\right] .
\end{aligned}
$$

2. Friedrichs symmetrizer. By $(\mathrm{H} 4)$ there exists a Friedrichs symmetrizer $s(V)$ for the $a^{j}(V)$. Using (3.5) we see that $s(V) M^{-1}(V)$ is a symmetrizer in $x \geq 0$ for the $\tilde{a}^{j}$, $j=0, \ldots, d-1$ and for $\tilde{\mathcal{A}}^{d}$. Thus, parallel to (7.14) we may now define $v_{2}$ on $\mathcal{O}$ by

$$
\begin{aligned}
& \mathbb{A}\left(\tilde{v}+v_{2}, 0\right) D\left(\tilde{v}+v_{2}\right)=0 \\
& \left.v_{2}\right|_{t=0}=v_{0},
\end{aligned}
$$

after taking appropriate extensions as before into $x<0 ; v_{1}$ is defined similarly.

3. $L^{2}$ estimate. Let $\tilde{H}\left(q_{ \pm}, \hat{\zeta}\right)$ and $\hat{\mathcal{B}}\left(q_{+}^{0}, q_{-}^{0} ; \hat{\zeta}\right)$ be the frozen coefficient operators obtained from $(8.7)$ just as $\mathcal{G}_{1}\left(q_{ \pm}, \hat{\zeta}\right)$ and $\hat{\mathcal{B}}\left(q_{+}^{0}, q_{-}^{0} ; \hat{\zeta}\right)$ in the ZND problem were obtained from (7.43). Now $w_{v N}$ in (7.49) is replaced by $\tilde{v}$. Also, let $H\left(q_{ \pm}, \hat{\zeta}\right)$ be defined just like $\tilde{H}\left(q_{ \pm}, \hat{\zeta}\right)$, but where $\tilde{a}^{j}$ is replaced by $a^{j}$ for all $j$. Since $\tilde{a}^{j}=a^{j}$ in $x \leq 0$ and $\tilde{a}^{j}=M a^{j}$ (3.5) in $x \geq 0$ we have

$$
\tilde{H}\left(q_{ \pm}, \hat{\zeta}\right)=H\left(q_{ \pm}, \hat{\zeta}\right)
$$


As before hypotheses (H0), (H1), and (H3) imply that for some $\delta_{0}>0$, the block structure condition is satisfied by the matrices $H\left(q_{ \pm}, \hat{\zeta}\right)$ for

$$
\left|q_{+}, q_{-}, q_{+}^{0}, q_{-}^{0}\right| \leq \delta_{0}
$$

Hence the same is true for $\tilde{H}\left(q_{ \pm}, \hat{\zeta}\right)$.

Similarly, since the decaying generalized eigenspaces for $\tilde{H}\left(q_{ \pm}, \hat{\zeta}\right)$ are the same as those for $H\left(q_{ \pm}, \hat{\zeta}\right)$, we conclude by continuity that nonvanishing of $\Delta_{C J}$ implies for $\delta_{0}$ small enough that the frozen problem defined by $\left(\tilde{H}\left(q_{ \pm}, \hat{\zeta}\right), \hat{\mathcal{B}}\left(q_{+}^{0}, q_{-}^{0} ; \hat{\zeta}\right)\right)$ satisfies the uniform Lopatinski condition. The $L^{2}$ estimate (7.42) now follows as before.

4. The remaining arguments are identical to those in the ZND case.

\section{References}

[Al] S.Alinhac, Existence d'ondes de raréfaction pour des sytèmes quasi-linéaires hyperboliques multidimensionnels, Comm. Partial Diff.Equ., 14 (1989), 173-230.

[BE] Barmin, A.A. and Egorushkin, S.A., Stability of shock waves, Adv. Mech. 15 (1992), no. $1-2,3-37$.

[CF] Courant, R. and Friedrichs, K., Supersonic flow and shock waves, Springer-Verlag, New York, 1976.

[Cos] Costanzino, N., Existence of curved multidimensional ZND fronts, Ph.D. Thesis, UNC Chapel Hill, 2006.

[CP] Chazarain, J. and Piriou, A., Introduction to the theory of linear partial differential equations, Studies in Mathematics and its Applications, 14, North-Holland, Amsterdam-New York, 1982. .

[CW1] Campbell, C. and Woodhead, D.W., The ignition of gases by an explosion wave I: carbon monoxide and carbon mixtures, J. Chem. Soc. 129 (1926), 3010-3021.

[CW2] Campbell, C. and Woodhead, D.W., Striated photographic records of explosion waves, J. Chem. Soc. 130 (1927), 1572-1578.

[Dy] D'yakov, A.P., On the stability of shock waves, (Russian) Z. Eksper. Teoret. Fiz. 27, (1954). 288-295.

[E1] Erpenbeck, J.J., Stability of steady-state equilibrium detonations, Physics of Fluids, 5, 1962, 604-614.

[E2] Erpenbeck, J.J., Stability of step shocks, Physics of Fluids, 5, 1962, 1181-1187.

[E3] Erpenbeck, J.J., Detonation stability for disturbances of small transverse wavelength, Physics of Fluids, 9, 1966, 1293-1306.

[FD] Fickett, W. and Davis, W., Detonation: Theory and Experiment, Univ. California Press, Berkeley, 1979. 
[G] Guès, O., Developpements asymptotiques de solutions exactes de systemes hyperboliques quasilineaires, Asymp. Analy. 6. 1993, pp.241-270.

[GS] Gasser, I. and Szmolyan, P., A geometric singular perturbation analysis of detonation and deflagration waves, SIAM J. Math. Anal., 24, 1993, 968-986.

[GS2] Gasser, I. and Szmolyan, P.,Detonation and deflagration waves with multistip reaction schemes, SIAM J. Applied Math.,55,175-191,1995.

[GMWZ] Guès, O., Métivier, G., Williams, M., and Zumbrun, K., Navier-Stokes regularization of multidimensional Euler shocks, to appear in Ann. Scient. ENS.

[GZ] Gardner, R. and Zumbrun, K., The gap lemma and geometric criteria instability of viscous shock profiles, Comm. Pure Appl. Math. 51. 1998, 797-855.

[HZ] Humpherys, J. and Zumbrun, K., A fast algorithm for numerical stability analysis of detonation waves in ZND, preprint, 2005.

[JL] Jenssen, H.K., and Lyng, G., The Lopatinski condition for gas dynamics, appendix (pp. 507-524) to K. Zumbrun, Stability of large-amplitude shock waves of compressible Navier-Stokes equations, 311-533, Handbook of Fluid Mechanics, Vol. III, North Holland, Amsterdam, 2004.

[JLW] Jenssen, H.K., Lyng, G., and Williams, M., Equivalence of low-frequency stability conditions for multidimensional detonations in three models of combustion, Indiana Univ. Math. J. 54 (2005), 1-64.

[K] Kreiss, H.O., Initial boundary value problems for hyperbolic systems, Comm. Pure Appl. Math. 23 (1970) 277-298.

[Ka] Kato, T., Perturbation Theory for Linear Operators, Springer-Verlag, Berlin 1985.

[LS] Lee, H. and Stewart, D., Calculation of linear detonation instability: one dimensional instability of plane detonation, J. Fluid Mech., 216,1990, 103-132.

[Ma1] Majda, A., The stability of multi-dimensional shock fronts - a new problem for linear hyperbolic equations. Mem. Amer. Math. Soc., No. 275, AMS, Providence, 1983.

[Ma2] Majda, A., The existence of multidimensional shock fronts, Mem. Amer. Math. Soc. No. 281, AMS, Providence, 1983.

[Ma3] Compressible fluid flow and systems of conservation laws in several space variables, Springer-Verlag, New York, 1984.

[MaZ] Mascia, C. and Zumbrun, K., Pointwise Green function bounds for shock profiles with degenerate viscosity, Arch. Rat. Mech. Anal. 169 (2003), 177-263.

[Met1] Metivier, G., Stability of multidimensional shocks, Advances in the theory of shock waves, Progress in Nonlinear PDE, 47, Birkhäuser, Boston, 2001. 
[Met2] Metivier, G., The block structure condition for symmetric hyperbolic systems, Bull. Lond. Math. Soc. 32. (2000), 689-702.

[MR] Majda, A. and Rosales, R., A theory for spontaneous Mach stem formation in reacting shock fronts, I. The basic perturbation analysis, SIAM J. Appl. Math., 43, 1983, 1310-1334.

[MZ1] Métivier, G. and Zumbrun, K., Large viscous boundary layers for noncharacteristic nonlinear hyperbolic problems, Mem. Amer. Math. Soc. 175 (2005), no. 826, vi+107 pp.

[MZ2] Métivier, G. and Zumbrun, K., Symmetrizers and continuity of stable subspaces for parabolic-hyperbolic boundary value problems, Disc. Cont. Dyn. Syst., 11, 2004, 205-220.

[Sh] Short, M., Multidimensional linear stability of a detonation wave at high activation energy, SIAM J. Appl. Math., 57,1997, 307-326.

[SS] Short, M. and Stewart, D.S., The multidimensional stability of weak heat-release detonations, J. Fluid Mech., 382, 1999, 109-135.

[S] Steenrod, N., The topology of fibre bundles, Princeton Univ. Press, Princeton, 1951.

[Wi] Williams, F., Combustion Theory, Benjamin-Cummings, Menlo Park, 1985.

[Z1] Zumbrun, K., Multidimensional stability of planar viscous shock waves, Advances in the theory of shock waves, 304-516. Progress in Nonlinear PDE, 47, Birkhäuser, Boston, 2001.

[Z2] Zumbrun, K., Stability of large-amplitude shock waves of compressible Navier-Stokes equations, Handbook of Mathematical Fluid Dynamics III, S. Friedlander and D. Serre eds., North-Holland, Amsterdam, 2004. 